\title{
Strong anomaly and phases of chiral gauge theories
}

\author{
Stefano Bolognesi, ${ }^{a, b}$ Kenichi Konishi ${ }^{a, b}$ and Andrea Luzio ${ }^{b, c}$ \\ ${ }^{a}$ Department of Physics "E. Fermi", University of Pisa, \\ Largo Pontecorvo, 3, Ed. C, 56127 Pisa, Italy \\ ${ }^{b}$ INFN - Sezione di Pisa, \\ Largo Pontecorvo, 3, Ed. C, 56127 Pisa, Italy \\ ${ }^{c}$ Scuola Normale Superiore, \\ Piazza dei Cavalieri, 7, 56127 Pisa, Italy \\ E-mail: stefano.bolognesi@unipi.it, kenichi.konishi@unipi.it, \\ andrea.luzio@sns.it
}

ABSTRACT: We present a simple argument which seems to favor, when applied to a large class of strongly-coupled chiral gauge theories, a dynamical-Higgs-phase scenario, characterized by certain bifermion condensates. Flavor symmetric confining vacua described in the infrared by a set of baryonlike massless composite fermions saturating the conventional 't Hooft anomaly matching equations, appear instead disfavored. Our basic criterion is that it should be possible to write a strong-anomaly effective action, analogous to the one used in QCD to describe the solution of the $\mathrm{U}(1)_{A}$ problem in the low-energy effective action, by using the low-energy degrees of freedom in the hypothesized infrared theory. We also comment on some well-known ideas such as the complementarity and the large $N$ planar dominance in the context of these chiral gauge theories. Some striking analogies and contrasts between the massless QCD and chiral gauge theories seem to emerge from this discussion.

KEywords: Anomalies in Field and String Theories, Effective Field Theories, Spontaneous Symmetry Breaking

ARXiv EPrint: 2105.03921 


\section{Contents}

1 Introduction 1

2 Nambu-Goldstone (NG) bosons and condensates 4

$2.1 N_{\mathrm{f}}$-flavored QCD 4

2.2 NonAbelian NG bosons in the $\psi \eta$ model 5

2.2.1 Colored $\psi \eta$ NG bosons 6

$2.3 \mathrm{U}(1) \mathrm{NG}$ boson(s) in the $\psi \eta$ model 7

$2.4 \mathrm{U}(1)$ symmetries in the $\chi \eta$ model 9

3 Strong anomaly and effective Lagrangian 11

3.1 Strong anomaly, U(1) problem and the $\theta$ dependence in QCD 11

3.1.1 Veneziano-Yankielowicz and Affleck-Dine-Seiberg superpotentials 12

3.2 Strong anomaly and effective action in the $\psi \eta$ model 12

3.3 Strong anomaly effective action in the generalized BY models 16

$\begin{array}{ll}3.4 & \text { Strong anomaly in the } \chi \eta \text { model } \\ \end{array}$

$\begin{array}{ll}\text { 3.5 Strong anomaly in the generalized GG models } & 18\end{array}$

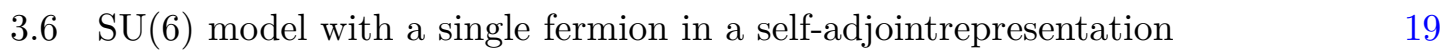

3.7 Adjoint QCD with $N_{\mathrm{c}}=N_{\mathrm{f}}=2 \quad 20$

4 Complementarity in the $\chi \eta$ model? $\quad 21$

5 Large $N$ planar dominance and U(1) NG bosons in chiral gauge theories 22

$6 \quad$ Multifield versus bi-fermion condensates 23

$\begin{array}{lll}7 & \text { Summary and discussion } & 26\end{array}$

$\begin{array}{ll}\text { A Chirally symmetric phase in the } \psi \boldsymbol{\eta} \text { model } & 29\end{array}$

B Color-flavor locked Higgs phase in the $\psi \eta$ model $\quad 29$

C Chirally symmetric phase in the $\chi \eta$ model 30

D Color-flavor locked Higgs vacuum in the $\chi \eta$ model 30

E Confining phase with unbroken global symmetries of the BY models 31

F Dynamical Higgs phase in the BY models 32

G Confining phase with unbroken global symmetries of the GG models $\quad 34$

H Dynamical Higgs phase in the generalized GG models 35 


\section{Introduction}

Our comprehension of the dynamics of strongly-coupled chiral gauge theories is still largely unsatisfactory, in spite of their potential role in describing the physics of fundamental interactions beyond the standard model. The results of many years of study of these theories, by using mixture of wisdom and certain consistency conditions, the most significant among them being 't Hooft's anomaly matching constraints [1], do restrict possible lists of dynamical scenarios and symmetry realization patterns [2]-[17], but they are usually far from being capable to determine the infrared physics of these systems uniquely.

It is customary in these discussions of the dynamics and symmetry realization in chiral gauge theories, to take into account the nonanomalous symmetries only. For instance, the 't Hooft anomaly matching requirements are normally applied exclusively on nonanomalous global symmetries.

From the point of view of renormalization group, assuming that the color interactions become strongly coupled towards the infrared, and that the low-energy effective degrees of freedom are not the original gluons and quarks (and similar color gauge bosons and matter fermions in general chiral theories), the anomalies present in the underlying theory must be reproduced for consistency either by composite massless fermions ('t Hooft) if the symmetry remains unbroken, or, if spontaneously broken, by massless Nambu-Goldstone (NG) bosons. In the latter case, the condition of "anomaly matching" is nothing but the well-known procedure for calculating the amplitudes (such as $\pi \rightarrow \gamma \gamma$, or much more generally, all anomalous amplitudes included in Wess-Zumino-Witten effective action) containing the NG bosons.

A third type of the application of renormalization-group invariance of anomaly concerns certain $\mathrm{U}(1)$ symmetry(ies), which is (are) affected by the topologically nontrivial gauge field configurations. This phenomenon goes under the name of strong anomaly, and the "U $(1)_{A}$ problem" and its solution in QCD $[18,19]$ is a renowned example in which the strong anomaly plays the central role. Probably because this appeared in the context of some characteristic aspects of QCD which is a vectorlike gauge theory, such as the chiral symmetry breaking and the low-energy sigma models, a similar question has not been discussed much in the context of strongly-coupled chiral gauge theories, to the best of our knowledge, with an exception being [5].

The aim of the present note is to discuss the implication of strong anomaly in stronglycoupled chiral gauge theories. Rather surprisingly, the requirement that the assumed set of infrared effective degrees of freedom (massless NG bosons and/or massless composite fermions à la 't Hooft) should be able to describe the strong-anomaly effective action in a way analogous to the famous strong-anomaly QCD effective action [20]-[24] containing certain logarithmic function, yields a rather solid indication about which type of the infrared phase is more plausible than others.

The models we will discuss in some details are the so-called Bars-Yankielowicz (BY) and generalized Georgi-Glasow (GG) models. In particular, our discussions will be set up first by using two simplest classes of models. One is an $\mathrm{SU}(N)$ gauge theory with 
left-handed fermions in the reducible, complex representation,

$$
\square \oplus(N+4) \bar{\square}
$$

that is,

$$
\psi^{\{i j\}}, \quad \eta_{i}^{B}, \quad i, j=1,2, \ldots, N, \quad B=1,2, \ldots, N+4,
$$

which is the simplest of the so-called Bars-Yankielowicz models [4]. This model will be called " $\psi \eta$ " model below, for short. ${ }^{1}$ The global symmetry group (actually the local property of the symmetry group) is

$$
G_{\mathrm{f}}=\mathrm{SU}(N+4) \times \mathrm{U}(1)_{\psi \eta},
$$

where $\mathrm{U}(1)_{\psi \eta}$ indicates the anomaly-free combination of $\mathrm{U}(1)_{\psi}$ and $\mathrm{U}(1)_{\eta}$, associated with the two types of matter Weyl fermions of the theory. Another model we consider is an $\mathrm{SU}(N)$ gauge theory with fermions

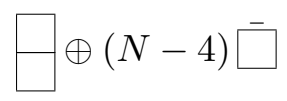

that is,

$$
\chi^{[i j]}, \quad \eta_{i}^{B}, \quad i, j=1,2, \ldots, N, \quad B=1,2, \ldots, N-4
$$

(the simplest Georgi-Glashow model). We will refer to it as " $\chi \eta$ " model below. The global symmetry group of the $\chi \eta$ model is

$$
G_{\mathrm{f}}=\mathrm{SU}(N-4) \times \mathrm{U}(1)_{\chi \eta} .
$$

Our interest is to understand how these symmetries are realized in the infrared.

More general Bars-Yankielowicz and Georgi-Glasow models, which are similar to the above two models but with $p$ additional pairs of fermions in the fundamental and antifundamental representations, will also be considered.

In all these models the conventional 't Hooft anomaly matching discussion apparently allows a confining phase, with no condensates and with full unbroken global symmetry, with some simple set of massless composite fermions saturating the anomaly matching equations. See appendix A for the $\psi \eta$ model, appendix $\mathrm{C}$ for the $\chi \eta$ model and appendices $\mathrm{E}$ and $\mathrm{G}$ for more general classes of BY and GG chiral gauge theories.

At the same time, the anomaly constraints are also consistent with a dynamical Higgs phase, in which the color and (part of) the flavor symmetry are dynamically broken by certain bifermion condensates, see appendices B, D, F and $\mathrm{H}$ (taken from [25]). These results are mostly known from the earlier work [4]-[17] and partly completed by ourselves, but are recorded here to make our discussion self-contained.

\footnotetext{
${ }^{1}$ In some earlier literature these fields were denoted by $S$ (the symmetric tensor $\psi$ ), $A$ (the antisymmetric tensor $\chi$ ), and $\bar{F}$ (the antifundamental, $\eta$ ).
} 
There is an aspect of the conventional 't Hooft anomaly matching procedure in the dynamical Higgs phase, which is perhaps not widely appreciated. The Higgs phase of these chiral theories are, in general, described by massless NG bosons as well as some massless fermions. These fermions saturate the conventional 't Hooft anomaly triangles with respect to the unbroken flavor symmetries. The way they do is, however, quite remarkable, and in our view, truly significant. As can be seen from table 2, table 4, and in similar tables 8, 9, 12 and 13 for the generalized BY and GG models (see appendices B, D, F, H), the set of fermions remaining massless in UV and those in the IR are identical as regards their quantum numbers, charges, and multiplicities. Therefore, the matching of anomalies (in the unbroken global symmetries) is completely automatic, and is natural. No arithmetic equations need to be solved to justify a solution which might look sometimes miraculous. The significance of such a solution of 't Hooft's equations (the dynamical Higgs phase) is its stablity. ${ }^{2}$

As we will find out below, the consideration of the strong anomaly appears to give us a rather clear indication that this second type of vacua - those in dynamical Higgs phase, characterized by certain bifermion condensates breaking color and part of the flavor symmetry - describe correctly the infrared dynamics of these chiral gauge theories.

This paper is organized as follows. In order to present the discussion systematically, we first review (for QCD) and work out for the $\psi \eta$ and $\chi \eta$ theories, the chiral Ward-Takahashi identities and identify the interpolating fields for all NG bosons, assuming the dynamical Higgs phase for these models (appendices B and D). This is done in section 2.

After this preparation, in section 3 we write the strong-anomaly low-energy effective action for the chiral gauge theories, $\psi \eta, \chi \eta$, as well as all other BY and GG models, following the procedure used for QCD. This discussion confirms the consistency of the dynamical Higgs phase of these models, in agreement with the recent mixed-anomaly study in these chiral gauge theories $[25,26]$, but it actually somewhat strengthens the conclusion. ${ }^{3}$

The basic observation is that there is no way of writing the strong-anomaly effective action if confining flavor symmetric vacua are assumed, i.e., by using only the massless baryons (appendices A, C, E, and G). The fermion-zero-mode (in the instanton background) counting would not work, in any of the chiral theories considered.

We shall comment briefly on some other types of theories (other than BY and GG models) as well, and discuss the implication of the strong anomaly on their phases.

Related questions regarding apparent complementarity in (only) one of the models (the $\chi \eta$ model) and a large $N$ argument concerning $\mathrm{U}(1)$ NG bosons in some chiral gauge theories, will be briefly commented upon in section 4 and in section 5 , respectively.

In section 7, our results are summarized. From the analyses of section $2 \sim$ section 5 some striking analogies and at the same time contrasts, between vectorlike theories and

\footnotetext{
${ }^{2}$ As noted in [25, 26], in the study making use of generalized anomalies one might try to make further gauging and study associated anomalies in the low-energy effective theory. From the identities of the sets of massless fermions in the UV and IR, it is seen that such an extra gauging would not produce any new unmatched anomalies.

${ }^{3}$ For instance the argument based on strong anomaly does not depend on whether $N$ is even or odd, whereas the mixed-anomaly calculation of $[25,26]$ were done for models with even $N$.
} 
chiral gauge theories seem to emerge. Better stated, one perhaps learns from these discussions a more precise meaning of what is similar or what is dissimilar between the strong interaction dynamics of vectorlike and chiral gauge theories beyond certain loose use of terminologies; as a result one gets a somewhat clearer understanding of the dynamics of strongly-coupled chiral gauge theories than before.

\section{Nambu-Goldstone (NG) bosons and condensates}

Consider any global continuous symmetry $G_{\mathrm{f}}$ and the associated conserved current $J_{\mu}$ and charge $Q$, the field $\phi$ (elementary or composite) which condenses and breaks $G_{\mathrm{f}}$, and the field $\tilde{\phi}$ which is transformed into $\phi$ by the $G_{\mathrm{f}}$ transformation:

$$
Q \equiv \int d^{3} x J_{0}, \quad[Q, \tilde{\phi}]=\phi, \quad\langle\phi\rangle \neq 0 .
$$

Thus

$$
\begin{aligned}
\lim _{q_{\mu} \rightarrow 0} i q^{\mu} \int d^{4} x e^{-i q \cdot x}\left\langle 0\left|T\left\{J_{\mu}(x) \tilde{\phi}(0)\right\}\right| 0\right\rangle & =\lim _{q_{\mu} \rightarrow 0} \int d^{4} x e^{-i q \cdot x} \partial_{\mu}\left\langle 0\left|T\left\{J_{\mu}(x) \tilde{\phi}(0)\right\}\right| 0\right\rangle \\
& =\int d^{3} x\left\langle 0\left|\left[J_{0}(x), \tilde{\phi}(0)\right]\right| 0\right\rangle \\
& =\langle 0|[Q, \tilde{\phi}(0)]| 0\rangle=\langle 0|\phi(0)| 0\rangle \neq 0
\end{aligned}
$$

This Ward-Takahashi like identity implies that the two-point function

$$
\int d^{4} x e^{-i q \cdot x}\left\langle 0\left|T\left\{J_{\mu}(x) \tilde{\phi}(0)\right\}\right| 0\right\rangle
$$

is singular at $q^{\mu} \rightarrow 0$. If the $G_{\mathrm{f}}$ symmetry is broken spontaneously such a singularity is due to a massless scalar particle in the spectrum -Nambu-Goldstone (NG) boson-, a "pion" below, symbolically, such that

$$
\left\langle 0\left|J_{\mu}(q)\right| \pi\right\rangle=i q_{\mu} F_{\pi}, \quad\langle\pi|\tilde{\phi}| 0\rangle \neq 0 .
$$

Two point function (2.3) (times $q^{\mu}$ ) behaves as

$$
\lim _{q^{\mu} \rightarrow 0} q^{\mu} \cdot q_{\mu} \frac{F_{\pi}\langle\pi|\tilde{\phi}| 0\rangle}{q^{2}} \sim \text { const } .
$$

The constant $F_{\pi}$ represents the amplitude for the broken current to produce the pion from the vacuum (the pion decay constant).

\section{$2.1 \quad N_{\mathrm{f}}$-flavored QCD}

In the standard QCD with $N_{\mathrm{f}}$ light flavors, the quarks are

$$
\psi_{L}^{i}, \psi_{R}^{i}, \quad i=1,2, \ldots, N_{\mathrm{f}} .
$$

We take

$$
\phi=\bar{\psi}_{R} \psi_{L}+\text { h.c. }, \quad \tilde{\phi}=\bar{\psi}_{R} t^{b} \psi_{L}-\text { h.c. } ; \quad J_{\mu}^{5, a}=i \bar{\psi}_{L} \bar{\sigma}_{\mu} t^{a} \psi_{L}-(L \leftrightarrow R),
$$


where $t^{a}$ is the $\mathrm{SU}\left(N_{\mathrm{f}}\right)$ generators, $a=1,2, \ldots N_{\mathrm{f}}^{2}-1$. It is believed, and confirmed by lattice simulations, that for sufficiently small $N_{\mathrm{f}}$, the field $\phi$ condenses,

$$
\langle\phi\rangle=\left\langle\bar{\psi}_{R} \psi_{L}+\text { h.c. }\right\rangle \sim-\Lambda^{3},
$$

leaving $\mathrm{SU}\left(N_{\mathrm{f}}\right)_{V} \times \mathrm{U}(1)_{V}$ unbroken. The axial $\mathrm{SU}\left(N_{\mathrm{f}}\right)_{A}$ is spontaneously broken:

$$
\left\langle\left[Q_{5}^{a}, \tilde{\phi}^{b}\right]\right\rangle=-\delta^{a b}\left\langle\bar{\psi}_{R} \psi_{L}+\bar{\psi}_{L} \psi_{R}\right\rangle=c \delta^{a b} \Lambda^{3} \neq 0,
$$

where $c$ is a constant of the order of unity. The axial $\mathrm{U}(1)_{A}$ is also spontaneously broken, but due to the strong anomaly the associated NG boson gets mass (the $\mathrm{U}(1)_{A}$ problem: see section 3.1).

\subsection{NonAbelian NG bosons in the $\psi \eta$ model}

In the dynamical Higgs phase of the $\psi \eta$ model (appendix B) the nonanomalous symmetry is broken as

$$
\mathrm{SU}(N) \times \mathrm{SU}(N+4) \times \mathrm{U}(1)_{\psi \eta} \stackrel{\langle\psi \eta\rangle}{\longrightarrow} \mathrm{SU}(N)_{\mathrm{cf}} \times \mathrm{SU}(4) \times \mathrm{U}(1)^{\prime} .
$$

Let us first concentrate our attention to $8 N$ NG bosons associated with the $\mathrm{SU}(N+4)$ breaking, leaving the discussion of the $\mathrm{U}(1) \mathrm{NG}$ boson and an unbroken $\mathrm{U}(1)^{\prime}$ symmetry to the next subsection. $\mathrm{An} \mathrm{SU}(N+4)$ current is $\left(T^{a}\right.$ is an $\mathrm{SU}(N+4)$ generator)

$$
J_{\mu}^{a}=i \bar{\eta}^{j, m} \bar{\sigma}_{\mu}\left(T^{a}\right)_{m n} \eta_{j}^{n}, \quad m, n=1,2, \ldots N+4
$$

and the charges are

$$
Q^{a}=\int d^{3} x J_{0}^{a}
$$

In particular, consider the broken symmetry currents ( $8 N$ components):

$$
J_{\mu}^{a}=i \bar{\eta}^{j, m} \bar{\sigma}_{\mu}\left(T^{a}\right)_{m n} \eta_{j}^{n},
$$

with

$$
m=1,2, \ldots N, \quad n=N+1, \ldots N+4, \quad \text { or vice versa },
$$

with charges

$$
Q^{a}=\int d^{3} x J_{0}^{a}=\int d^{3} x \bar{\eta}^{j, m}\left(T^{a}\right)_{m n} \eta_{j}^{n} .
$$

The associated symmetry is broken by the condensates

$$
\left\langle\psi^{\{i j\}} \eta_{j}^{m}\right\rangle=c_{\psi \eta} \Lambda^{3} \delta^{i m}, \quad i, m=1,2, \ldots N
$$

and there will be $8 N$ NG bosons associated with the currents, $J_{\mu}^{a}$. A natural choice for the pion interpolating field is a gauge-invariant composite,

$$
\phi^{\tilde{a}}=\left(\psi^{i k} \eta_{k}^{n}\right)^{*}\left(T^{\tilde{a}}\right)_{n m}\left(\psi^{i j} \eta_{j}^{m}\right) .
$$


The relevant commutators are:

$$
\left\langle\left[Q^{a}, \phi^{\tilde{a}}\right]\right\rangle=-\left[T^{a}, T^{\tilde{a}}\right]_{m n}\left\langle\left(\psi^{i k} \eta_{k}^{m}\right)^{*}\left(\psi^{i j} \eta_{j}^{n}\right)\right\rangle .
$$

Let us consider various $\mathrm{SU}(2)$ subalgebras $\left(\sigma^{i}\right.$ 's are the Pauli matrices)

$$
T^{a}=\frac{\sigma^{1}}{2} \quad T^{\tilde{a}}=\frac{\sigma^{2}}{2}, \quad T^{0}=\frac{\sigma^{3}}{2}
$$

living in the $2 \times 2$ subspace $(m, n)$,

$$
m \in\{1,2, \ldots N\}, \quad n \in\{N+1, \ldots N+4\},
$$

for the broken charge $Q^{a}(2.15)$ and the pion field $\phi^{\tilde{a}}$ (2.17), respectively. The commutator (2.18) now gives

$$
\left\langle\left[Q^{a}, \phi^{\tilde{a}}\right]\right\rangle=-\frac{1}{2}\left\langle\left(\psi^{i k} \eta_{k}^{m}\right)^{*}\left(\psi^{i j} \eta_{j}^{m}\right)\right\rangle+\frac{1}{2}\left\langle\left(\psi^{i k} \eta_{k}^{n}\right)^{*}\left(\psi^{i j} \eta_{j}^{n}\right)\right\rangle
$$

( no summation over $m$ or $n$ here and below, in (2.24)). ${ }^{4}$ Upon condensation of color-flavor diagonal form (2.16), the above becomes

$$
\left\langle\left[Q^{a}, \phi^{\tilde{a}}\right]\right\rangle=-\frac{1}{2}\left(c_{\psi \eta} \Lambda^{3}\right)^{2} \neq 0,
$$

implying that $Q^{a}$ generates a massless NG bosons from the vacuum, whose "wave function" is roughly

$$
\left\langle 0\left|\phi^{\tilde{a}}(x)\right| \pi^{a}(p)\right\rangle=f_{p}(x) .
$$

Note that, by inserting (2.16), the pion interpolating field (2.17) can also be written in a simpler, gauge dependent form:

$$
\phi^{\tilde{a}} \sim\left(\psi^{m k} \eta_{k}^{n}\right)^{*}\left(T^{\tilde{a}}\right)_{n m}+\left(T^{\tilde{a}}\right)_{m n}\left(\psi^{m j} \eta_{j}^{n}\right)=2 \Re\left\{\left(T^{\tilde{a}}\right)_{m n}\left(\psi^{m j} \eta_{j}^{n}\right)\right\} .
$$

Clearly, the pairing between the charge and the pion field can be interchanged:

$$
T^{a}=\frac{\sigma^{2}}{2} \quad T^{\tilde{a}}=\frac{\sigma^{1}}{2}, \quad T^{0}=\frac{\sigma^{3}}{2}
$$

(instead of (2.19)); therefore one finds $4 N \times 2=8 N$ NG bosons.

A simpler way to state the result is, as can be seen by inspection of (2.19), (2.25), and (2.24), that the $8 N$ NG bosons of non diagonal $\frac{\mathrm{SU}(N+4)}{\mathrm{SU}(N) \times \mathrm{SU}(4)}$ generators are just the real and imaginary parts of $\psi^{m j} \eta_{j}^{n}(m \leq N, n \geq N+1)$.

\subsubsection{Colored $\psi \eta$ NG bosons}

The $N^{2}-1$ colored NG bosons

$$
\begin{aligned}
\tilde{\phi}^{b} & \sim\left(\psi^{m k} \eta_{k}^{n}\right)^{*}\left(T^{b}\right)_{n m}+\left(T^{b}\right)_{n m}\left(\psi^{n j} \eta_{j}^{m}\right), \\
& =\left(\psi^{n k} \eta_{k}^{m}\right)^{*}\left(T^{b}\right)_{m n}+\left(T^{b}\right)_{n m}\left(\psi^{n j} \eta_{j}^{m}\right), \\
& \sim \Re\left\{\left(T^{b}\right)_{n m}\left(\psi^{n j} \eta_{j}^{m}\right)\right\}, \quad n \leq N, \quad m \leq N, \quad T^{b} \in \operatorname{su}(N),
\end{aligned}
$$

generated by the condensates (2.16) are absorbed by the $\mathrm{SU}(N)$ gauge bosons by the Englert-Brout-Higgs mechanism, so do not appear as physical massless particles.

\footnotetext{
${ }^{4}$ The summation over the repeated color indices, $j, k$ are done, as usual.
} 


\section{$2.3 \mathrm{U}(1)$ NG boson(s) in the $\psi \eta$ model}

Assuming again that we are in the dynamical Higgs phase of the $\psi \eta$ model, let us examine the fate of the two nonanomalous $\mathrm{U}(1)$ symmetries, $\mathrm{U}(1)_{\psi \eta}$ and a $\mathrm{U}(1)_{\mathrm{f}} \subset \mathrm{SU}(N+4)$. One combination is broken spontaneously, giving rise to a physical NG boson, and the other combination, $\mathrm{U}(1)^{\prime}$, remains unbroken, as a manifest symmetry of the low-energy theory.

For the $\mathrm{U}(1)_{\psi \eta}$ symmetry, writing the matter fermions together as

$$
\left(\begin{array}{c}
\psi \\
\eta
\end{array}\right)=\left(\begin{array}{c}
\psi^{\{i j\}} \\
\eta_{i}^{1} \\
\vdots \\
\eta_{i}^{N+4}
\end{array}\right)
$$

the $\mathrm{U}(1)_{\psi \eta}$ current is

$$
J^{\mu}=i(\bar{\psi} \bar{\eta}) T_{\psi \eta} \bar{\sigma}^{\mu}\left(\begin{array}{l}
\psi \\
\eta
\end{array}\right), \quad Q=\int d^{3} x J^{0}
$$

where

$$
T_{\psi \eta}=\left(\begin{array}{ll}
(N+4) \mathbf{1}_{\frac{N(N+1)}{2}} & \\
& -(N+2) \mathbf{1}_{(N+4) \cdot N}
\end{array}\right)
$$

and the $\mathrm{U}(1)_{\psi \eta}$ charge operator is

$$
Q_{\psi \eta}=\int d^{3} x\left[(N+4) \bar{\psi}_{i j} \psi^{i j}-(N+2) \sum_{m} \bar{\eta}_{m}^{i} \eta_{i}^{m}\right]
$$

so

$$
\begin{aligned}
& {\left[Q_{\psi \eta}, \psi^{k \ell} \eta_{\ell}^{n}\right]=(-(N+4)+N+2) \psi^{k \ell} \eta_{\ell}^{n}=-2 \psi^{k \ell} \eta_{\ell}^{n}} \\
& {\left[Q_{\psi \eta}, \bar{\psi}_{k \ell} \bar{\eta}_{n}^{\ell}\right]=(N+4-(N+2)) \bar{\psi}_{k \ell} \bar{\eta}_{n}^{\ell}=2 \bar{\psi}_{k \ell} \bar{\eta}_{n}^{\ell} .}
\end{aligned}
$$

The condensate is (2.16):

$$
\left\langle\psi^{i j} \eta_{j}^{m}\right\rangle=c_{\psi \eta} \delta^{i m} \Lambda^{3}, \quad i, m \leq N
$$

so

$$
\left\langle\left[Q_{\psi \eta}, \psi^{k \ell} \eta_{\ell}^{n}\right]\right\rangle \neq 0, \quad k=n=1,2, \ldots, N .
$$

On the other hand, a diagonal $\mathrm{SU}(N+4)$ generator (it acts only on $\eta$ ) which mixes with $\mathrm{U}(1)_{\psi \eta}$ is

with charge operator

$$
T_{\mathrm{f}}=\left(\begin{array}{ccc}
\mathbf{0}_{\frac{N(N+1)}{2}} & & \\
& 4 \mathbf{1}_{N \cdot N} & \\
& & -N \mathbf{1}_{4 \cdot N}
\end{array}\right),
$$

$$
\begin{aligned}
Q_{\mathrm{f}} & =\int d^{3} x\left[4 \sum_{m=1}^{N} \bar{\eta}_{m}^{i} \eta_{i}^{m}-N \sum_{m=N+1}^{N+4} \bar{\eta}_{m}^{i} \eta_{i}^{m}\right], \\
{\left[Q_{\mathrm{f}}, \psi^{k \ell} \eta_{\ell}^{n}\right] } & =\left\{\begin{array}{lll}
4 \psi^{k \ell} \eta_{\ell}^{n} & \text { for } & n \leq N ; \\
-N \psi^{k \ell} \eta_{\ell}^{n} & \text { for } & n>N .
\end{array}\right.
\end{aligned}
$$


The same condensate (2.32) breaks also $Q_{\mathrm{f}}$, as

$$
\left\langle\left[Q_{\mathrm{f}}, \psi^{k \ell} \eta_{\ell}^{n}\right]\right\rangle \neq 0, \quad k=n=1,2, \ldots, N
$$

But the combination $\mathrm{U}(1)^{\prime}$ generated by

$$
Q^{\prime}=2 Q_{\psi \eta}+Q_{\mathrm{f}}
$$

remains unbroken:

$$
\left\langle\left[2 Q_{\psi \eta}+Q_{\mathrm{f}}, \psi^{k \ell} \eta_{\ell}^{n}\right]\right\rangle=0 \quad(\forall k, \forall n)
$$

Any other combination of $Q_{\mathrm{f}}$ and $Q_{\psi \eta}$ is spontaneously broken, see eqs. (2.31), (2.36), so there is one physical U(1) NG boson in this model. A natural choice for the interpolating field for this physical U(1) NG boson would be

$$
\sum_{n, j}^{N} \psi^{n j} \eta_{j}^{n}=\operatorname{Tr}(\psi \eta) \propto \mathbf{1}+\frac{i}{F_{\pi}^{(0)}} \phi_{0}+\ldots
$$

where the field are appropriately normalized and $F_{\pi}^{(0)}$ is a constant with a mass dimension. This is an analogue of $\bar{\psi}_{R} \psi_{L}=\bar{u}_{R} u_{L}+\bar{d}_{R} d_{L}+\ldots$ in QCD, and also analogous to the nonAbelian NG bosons, (2.24).

Unlike $\bar{\psi}_{R} \psi_{L}$ in QCD, (2.40) is not gauge-invariant. However, it is not difficult to find a natural gauge-invariant form for the interpolating field for the same NG boson: it could be written as

$$
\operatorname{det} U, \quad U^{k \ell}=\psi^{k j} \eta_{j}^{\ell}
$$

by using the first $N$ flavors, $\eta_{j}^{a}, a=1,2, \ldots, N$. Note that this composite field is a singlet of the surviving symmetry of the $\psi \eta$ system, (2.10). This will play an important role in the discussion of the strong anomaly below.

Expanding around the VEV, (2.32), (2.40) and (2.41) give the same physical field,

$$
\sim \text { const }+\sum_{k, j}^{N}\left(\psi^{k j} \eta_{j}^{k}\right)^{\{q\}}
$$

where $\left(\psi^{k j} \eta_{j}^{k}\right)^{\{q\}}$ indicates the fluctuation part of the composite field, $\psi^{k j} \eta_{j}^{k}$.

So far, we considered two nonanomalous $\mathrm{U}(1)$ symmetries, $\mathrm{U}(1)_{\psi \eta}$ and a $\mathrm{U}(1)_{\mathrm{f}} \subset$ $\mathrm{SU}(N+4)$, and found that one combination remains a manifest symmetry, call it $\mathrm{U}(1)^{\prime}$, while the other (let us indicate as $\mathrm{U}(1)_{\mathrm{NG}}$ ) gets broken and generates an associated, physical massless NG boson.

Actually, the system possesses one more, independent, U(1) symmetry, though anomalous. Any combination of $\mathrm{U}(1)_{\psi}$ and $\mathrm{U}(1)_{\eta}$ other than $\mathrm{U}(1)_{\psi \eta}$ is anomalous (let us call it $\mathrm{U}(1)_{\mathrm{an}}$ ) hence cannot be expressed as a linear combination of $\mathrm{U}(1)^{\prime}$ and $\mathrm{U}(1)_{\mathrm{NG}}$.

It is perhaps useful to compare the situation here with the massless QCD vacuum, with quark condensate, (2.9). In the latter, there is one manifest symmetry, $\mathrm{U}(1)_{V}$, and an axial 
symmetry $\mathrm{U}(1)_{A}$ which is anomalous, and is also spontaneously broken (associated with a would-be NG boson which becomes massive by the strong anomaly, as reviewed below, section 3.1). In the $\psi \eta$ model under consideration, there is one manifest symmetry, $\mathrm{U}(1)^{\prime}$, one spontaneously broken, nonanomalous, $\mathrm{U}(1)_{N G}$, with an associated physical massless $\mathrm{NG}$ boson, and an anomalous $\mathrm{U}(1)_{\text {an }}$ symmetry, independent of $\mathrm{U}(1)^{\prime}$ and $\mathrm{U}(1)_{\mathrm{NG}}$. There is thus one more (counting also anomalous and/or spontaneously broken) U(1) symmetry in our model, as compared to the massless QCD.

It is tempting to regard the $\mathrm{U}(1)_{\text {an }}$ symmetry as an analogue of the $\mathrm{U}(1)_{A}$ symmetry in QCD. However, as it turns out, the nature of $\mathrm{U}(1)_{\text {an }}$ (whether or not it is spontaneously broken), and related to that question, the true form of the interpolating field for the physical massless NG boson ((2.40), (2.41), or something else), can only be found after an appropriate study of the strong anomaly effective action for the $\psi \eta$ system. This will be done in section 3.2. Somewhat surprisingly, it will be found that the dynamical Higgs phase of the $\psi \eta$ model is not exhaustively characterized by the condensate of $\operatorname{det} U$. The proper form of the strong-anomaly effective action leads to a second, bi-baryon type condensate besides $\langle\operatorname{det} U\rangle$, see (3.26). This turns out to be the missing piece of the puzzle. The true interpolating function for the physical massless $\mathrm{U}(1)_{\mathrm{NG}}$ boson will be found, not to be given by $\phi_{0}$ of (2.40), but by $\phi$ in (3.33).

\section{$2.4 \mathrm{U}(1)$ symmetries in the $\chi \eta$ model}

In the dynamical Higgs phase of the $\chi \eta$ model the symmetry breaking proceeds as

$$
\begin{aligned}
\mathrm{SU}(N) \times \mathrm{SU}(N-4) \times \mathrm{U}(1)_{\chi \eta} \stackrel{\langle\chi \eta\rangle}{\longrightarrow} & \mathrm{SU}(N-4)_{\mathrm{cf}} \times \mathrm{SU}(4)_{\mathrm{c}} \times \mathrm{U}(1)^{\prime} \\
\longrightarrow & \mathrm{SU}(N-4)_{\mathrm{cf}} \times \mathrm{U}(1)^{\prime},
\end{aligned}
$$

where the residual color $\mathrm{SU}(4)_{\mathrm{c}}$, unbroken by the condensates

$$
\left\langle\chi^{i j} \eta_{j}^{m}\right\rangle=c_{\chi \eta} \delta^{i m} \Lambda^{3}, \quad i, m=1,2, \ldots, N-4,
$$

evolves further towards infrared, confines, develops another condensate,

$$
\langle\chi \chi\rangle \neq 0
$$

and gives rise to a hidden (dark matter?) sector invisible to the massless sector.

For the $\mathrm{U}(1)_{\chi \eta}$ symmetry, writing the matter fermions together as

$$
\left(\begin{array}{c}
\chi \\
\eta
\end{array}\right)=\left(\begin{array}{c}
\chi^{[i j]} \\
\eta_{i}^{1} \\
\vdots \\
\eta_{i}^{N-4}
\end{array}\right)
$$

the $\mathrm{U}(1)_{\chi \eta}$ current is

$$
J^{\mu}=i(\bar{\chi} \bar{\eta}) T_{\chi \eta} \bar{\sigma}^{\mu}\left(\begin{array}{c}
\chi \\
\eta
\end{array}\right), \quad Q=\int d^{3} x J^{0},
$$


where

$$
T_{\chi \eta}=\left(\begin{array}{ll}
(N-4) \mathbf{1}_{\frac{N(N-1)}{2}} & \\
& -(N-2) \mathbf{1}_{(N-4) \cdot N}
\end{array}\right)
$$

and the charge operator is

$$
Q_{\chi \eta}=\int d^{3} x\left[(N-4) \bar{\chi}_{i j} \chi^{i j}-(N-2) \sum_{m} \bar{\eta}_{m}^{i} \eta_{i}^{m}\right],
$$

so

$$
\begin{aligned}
& {\left[Q_{\chi \eta}, \chi^{k \ell} \eta_{\ell}^{n}\right]=(-(N-4)+N-2) \psi^{k \ell} \eta_{\ell}^{n}=2 \chi^{k \ell} \eta_{\ell}^{n}} \\
& {\left[Q_{\chi \eta}, \bar{\chi}_{k \ell} \bar{\eta}_{n}^{\ell}\right]=(N-4-(N-2)) \bar{\chi}_{k \ell} \bar{\eta}_{n}^{\ell}=-2 \bar{\chi}_{k \ell} \bar{\eta}_{n}^{\ell}}
\end{aligned}
$$

The condensate (2.44) leads to

$$
\left\langle\left[Q_{\chi \eta}, \chi^{k \ell} \eta_{\ell}^{m}\right]\right\rangle \neq 0, \quad k=m=1,2, \ldots, N-4 .
$$

On the other hand, a diagonal color $\mathrm{U}(1)_{\mathrm{c}} \subset \mathrm{SU}(N)$ generator (which mixes with $\left.\mathrm{U}(1)_{\psi \eta}\right)$ is

$$
T_{\mathrm{c}}=\left(\begin{array}{ll}
4 \mathbf{1}_{N-4} & \\
& -(N-4) \mathbf{1}_{4}
\end{array}\right),
$$

with charge operator $Q_{\mathrm{c}}$

$$
\left[Q_{\mathrm{c}}, \chi^{k \ell} \eta_{\ell}^{n}\right]=\left\{\begin{array}{lll}
4 \chi^{k \ell} \eta_{\ell}^{n} & \text { for } & k \leq N-4 \\
-(N-4) \chi^{k \ell} \eta_{\ell}^{n} & \text { for } & k>N-4 .
\end{array}\right.
$$

The same condensate $(2.44)$ breaks both $\mathrm{U}(1)_{\mathrm{c}}$ and $\mathrm{U}(1)_{\chi \eta}$, but the combination $\mathrm{U}(1)^{\prime}$ generated by

$$
2 Q_{\chi \eta}-Q_{\mathrm{c}}
$$

remains unbroken:

$$
\left[2 Q_{\chi \eta}-Q_{\mathrm{c}}, \chi^{k \ell} \eta_{\ell}^{n}\right]=0
$$

Unlike in the $\psi \eta$ model, therefore, no physical massless NG bosons appear in the $\chi \eta$ model, as the potential NG boson is eaten up by the $\mathrm{U}(1)_{\mathrm{c}} \subset \mathrm{SU}(N)$ color gauge boson by the Englert-Brout-Higgs mechanism.

As in the $\psi \eta$ model, there is actually another $\mathrm{U}(1)$ symmetry (any combination of $\mathrm{U}(1)_{\chi}$ and $\mathrm{U}(1)_{\eta}$, other than $\left.\mathrm{U}(1)_{\chi \eta}\right)$. This $\mathrm{U}(1)$ is also spontaneously broken by the condensates, (2.44), but the associated pseudo-NG boson gets mass by strong anomaly, as in the $\mathrm{U}(1)_{A} \mathrm{NG}$ boson of QCD. The fact that there are no physical, massless NG bosons in the $\chi \eta$ model does not mean that this anomalous $\mathrm{U}(1)$ symmetry is unimportant for the discussion of the infrared dynamics, see the next section.

A closely related question is what is eventually the gauge-invariant form of the condensate, (2.44). A natural choice for the $\chi \eta$ model is $\left((\chi \eta)^{i m} \equiv \chi^{i j} \eta_{j}^{m}\right)$

$$
\begin{aligned}
U & =\epsilon_{i_{1} i_{2} \ldots i_{N}} \epsilon_{m_{1} m_{2} \ldots m_{N-4}}(\chi \eta)^{i_{1} m_{1}}(\chi \eta)^{i_{2} m_{2}} \ldots(\chi \eta)^{i_{N-4} m_{N-4}} \chi^{i_{N-3} i_{N-2}} \chi^{i_{N-1} i_{N}} \\
& \sim \epsilon(\chi \eta)^{N-4} \chi \chi .
\end{aligned}
$$




\section{Strong anomaly and effective Lagrangian}

In this section, we first review the well-known strong-anomaly effective action in QCD and then, following the same steps, write down the analogous effective action for chiral gauge theories.

\subsection{Strong anomaly, U(1) problem and the $\theta$ dependence in QCD}

In the discussion of the dynamics of QCD the consideration of the anomalous axial $\mathrm{U}(1)_{A}$ symmetry has been quite important, in relation to the so-called U(1) problem and its solution $[18,19]$. Even though the NG boson(s) associated with the anomalous $\mathrm{U}(1)_{A}$ $\left(\eta, \eta^{\prime}\right)^{5}$ get mass by the strong interaction dynamics $\left(m_{\eta} \gg m_{\pi}, m_{\eta^{\prime}} \gg m_{K}\right)$ the presence of the anomalous U(1) symmetry has a deep implication on the spontaneous breaking of the nonanomalous chiral symmetries,

$$
\mathrm{SU}\left(N_{\mathrm{f}}\right)_{L} \times \mathrm{SU}\left(N_{\mathrm{f}}\right)_{R} \rightarrow \mathrm{SU}\left(N_{\mathrm{f}}\right)_{V},
$$

which generates physical lightest NG bosons, the pions.

Such a logical connection is best seen in the effective Lagrangian approach for QCD in the large $N$ limit. Generalizing the standard sigma model Lagrangian to include the effect of strong anomaly, the authors of [20]-[24] write

$$
L=L_{0}+\hat{L},
$$

where $L_{0}$ is the standard sigma model effective Lagrangian

$$
L_{0}=\frac{F_{\pi}^{2}}{2} \operatorname{Tr} \partial_{\mu} U \partial^{\mu} U^{\dagger}+\operatorname{Tr} M U+\text { h.c. }+\ldots ; \quad U \equiv \bar{\psi}_{R} \psi_{L},
$$

and $\hat{L}$ represents the strong anomaly

$$
\hat{L}=\frac{i}{2} q(x) \log \operatorname{det} U / U^{\dagger}+\frac{N}{a_{0} F_{\pi}^{2}} q^{2}(x)-\theta q(x),
$$

$q(x)$ is the topological density

$$
q(x)=\frac{g^{2}}{32 \pi^{2}} F_{\mu \nu}^{a} \tilde{F}^{a, \mu \nu},
$$

$a_{0}$ is a constant of the order of unity, $F_{\pi}$ the pion decay constant, and $\theta$ is the QCD vacuum parameter. The $\mathrm{U}(1)_{A}$ anomaly under

$$
\Delta S=2 N_{\mathrm{f}} \alpha \int d^{4} x \frac{g^{2}}{32 \pi^{2}} F_{\mu \nu}^{a} \tilde{F}^{a, \mu \nu}, \quad \psi_{L} \rightarrow e^{i \alpha} \psi_{L}, \quad \psi_{R} \rightarrow e^{-i \alpha} \psi_{R}
$$

is reproduced by the $\log \operatorname{det} U / U^{\dagger}$ term of the effective action.

\footnotetext{
${ }^{5}$ Here $\eta, \eta^{\prime}$ are the singlet pseudoscalar mesons of the real world, as in the Particle Data Booklet. The attentive reader will not confuse them with the Weyl fermion in the chiral $\psi \eta$ or $\chi \eta$ models being studied in the present work.
} 
Treating $q(x)$ as an auxiliary field, and integrating, one gets another form of the anomaly term $[20-24]$

$$
\hat{L}=-\frac{F_{\pi}^{2} a_{0}}{4 N}\left(\theta-\frac{i}{2} \log \operatorname{det} U / U^{\dagger}\right)^{2}
$$

It has been noted that a multi-valued effective Lagrangian involving $\log \operatorname{det} U / U^{\dagger}$ is only well defined because

$$
\langle U\rangle \propto \mathbf{1} \neq 0:
$$

the effective potential is defined as its expansion around its VEV,

$$
U \propto e^{i \frac{\pi^{a} t^{a}}{F_{\pi}}+i \frac{\eta t^{0}}{F_{\pi}^{(0)}}}=\mathbf{1}+i \frac{\pi^{a} t^{a}}{F_{\pi}}+i \frac{\eta t^{0}}{F_{\pi}^{(0)}}+\ldots
$$

Inverting the logics, one might actually argue that the presence of such an effective action reproducing the strong anomaly implies a nonvanishing condensate, $\langle U\rangle=$ $\left\langle\bar{\psi}_{R} \psi_{L}\right\rangle \neq 0$, and hence indirectly the spontaneous breaking of nonanomalous chiral symmetry, $\mathrm{SU}\left(N_{\mathrm{f}}\right)_{L} \times \mathrm{SU}\left(N_{\mathrm{f}}\right)_{R} \rightarrow \mathrm{SU}\left(N_{\mathrm{f}}\right)_{V}$, affecting the low-energy physics.

\subsubsection{Veneziano-Yankielowicz and Affleck-Dine-Seiberg superpotentials}

In the context of $\mathcal{N}=1$ supersymmetric gauge theories, the strong-anomaly effective action is expressed by the so-called Veneziano-Yankielowicz (VY) and Affleck-Dine-Seiberg (ADS) superpotentials [27-29]. They correctly reproduce in the infrared effective theory the effects of instantons, supersymmetric Ward-Takahashi identities, and the anomaly of [30, 31]. The VY and ADS superpotentials are crucial in determinig the infrared dynamics and phases of the $\mathcal{N}=1$ supersymmetric gauge theories (see [32] for a review).

\subsection{Strong anomaly and effective action in the $\psi \eta$ model}

Unlike $\bar{\psi}_{R} \psi_{L}$ in QCD, $\tilde{\phi}=\sum_{n, j}^{N} \psi^{n j} \eta_{j}^{n}$ is not gauge invariant. This is not a problem if the system is assumed to be in dynamical Higgs phase of the $\psi \eta$ model, in which the low-energy symmetry is

$$
\mathrm{SU}(N)_{\mathrm{cf}} \times \mathrm{SU}(4) \times \mathrm{U}(1)^{\prime},
$$

(appendix B). A gauge invariant form of the condensate, consistent with such a symmetry is:

$$
\operatorname{det} U, \quad U^{k \ell} \equiv \psi^{k j} \eta_{j}^{\ell} .
$$

In terms of this composite field, one may write the low-energy effective Lagrangian describing the strong anomaly,

$$
\hat{L}=\frac{i}{2} q(x) \log \operatorname{det} U / U^{\dagger}, \quad q(x)=\frac{g^{2}}{32 \pi^{2}} F_{\mu \nu}^{a} \tilde{F}^{a, \mu \nu},
$$

which looks very much in analogy with the strong-anomaly effective action of QCD, (3.4). ${ }^{6}$ The multivalued, logarithmic potential is well defined, as we are in the dynamical Higgs

\footnotetext{
${ }^{6} \theta$ parameter is absent in chiral gauge theories.
} 
phase, $\langle U\rangle \propto \mathbb{1}$. As in QCD, one may actually reverse the logics and argue that the strong anomaly effective action, which should be present in the low-energy effective theory for faithfully representing all the symmetries of the UV theory, implies the nonvanishing condensates, $\langle\operatorname{det} U\rangle \neq 0$, which means the global symmetry breaking as in (3.10), i.e., the system is in Higgs phase.

Though (3.12) appears to be a natural choice in the broken phase, it has a defect of not being invariant under the full symmetry of the underlying theory,

$$
\mathrm{SU}(N) \times \mathrm{SU}(N+4) \times \mathrm{U}(1)_{\psi \eta} .
$$

A correct low-energy effective action should be invariant under the full symmetry, and must describe (at least, be consistent with) the breaking from (3.13) to (3.10). This observation brings us back to the questions on the nature of $\mathrm{U}(1)$ NG bosons, raised at the end of section 2.3 .

In order to get to the right form of the strong-anomaly effective action, we start from the beginning,

$$
\begin{aligned}
\mathcal{L} & =-\frac{1}{4} F_{\mu \nu} F^{\mu \nu}+\mathcal{L}^{\text {fermions }} \\
\mathcal{L}^{\text {fermions }} & \left.=-i \bar{\psi} \bar{\sigma}^{\mu}\left(\partial+\mathcal{R}_{\mathrm{S}}(a)\right)_{\mu} \psi-i \bar{\eta} \bar{\sigma}^{\mu}\left(\partial+\mathcal{R}_{\mathrm{F}^{*}}(a)\right)\right)_{\mu} \eta
\end{aligned}
$$

( $a$ is the $\mathrm{SU}(N)$ gage field, and the matrix representations appropriate for $\psi$ and $\eta$ fields are indicated in an obvious notation). Change the variables by

$$
\mathcal{L}=-\frac{1}{4} F_{\mu \nu} F^{\mu \nu}+\mathcal{L}^{\text {fermions }}+\operatorname{Tr}\left[(\psi \eta)^{*} U\right]+\text { h.c. }+B(\psi \eta \eta)^{*}+\text { h.c. },
$$

where $U$ is the composite scalars of $N \times(N+4)$ color-flavor mixed matrix form,

$$
\operatorname{Tr}\left[(\psi \eta)^{*} U\right] \equiv\left(\psi^{i j} \eta_{j}^{m}\right)^{*} U^{i m}
$$

and $B$ are the baryons $B \sim \psi \eta \eta$,

$$
B^{m n}=\psi^{i j} \eta_{i}^{m} \eta_{j}^{n},
$$

antisymmetric in $m \leftrightarrow n$. Here we have anticipated the fact that these baryonlike composite fields, present in the Higgs phase together with the composite scalars $\psi \eta$ (see appendix B), are also needed to write down the strong-anomaly effective action, see below.

Integrating over $\psi$ and $\eta$ one gets

$$
\mathcal{L}^{\mathrm{eff}}=-\frac{1}{4} F_{\mu \nu} F^{\mu \nu}+\operatorname{Tr}(\mathcal{D} U)^{\dagger} \mathcal{D} U-i \bar{B} \bar{\sigma}^{\mu} \partial_{\mu} B-V .
$$

The potential $V$ is assumed to be such that its minimum is of the form, (2.16):

$$
\left\langle U^{i m}\right\rangle=c_{\psi \eta} \Lambda^{3} \delta^{i m}, \quad i, m=1,2, \ldots N,
$$

and among other terms, it contains the strong anomaly term, $\hat{L}$,

$$
V=V^{(0)}+\hat{L},
$$


which we choose as

$$
\hat{L}=\text { const }\left[\log (\epsilon B B \operatorname{det} U)-\log (\epsilon B B \operatorname{det} U)^{\dagger}\right]^{2} .
$$

The argument of the logarithm

$$
\epsilon B B \operatorname{det} U \equiv \epsilon^{m_{1}, m_{2}, \ldots, m_{N+4}} \epsilon^{i_{1}, i_{2}, \ldots, i_{N}} B_{m_{N+1}, m_{N+2}} B_{m_{N+3}, m_{N+4}} U_{i_{1} m_{1}} U_{i_{2} m_{2}} \ldots U_{i_{N} m_{N}}
$$

is invariant under the full (nonanomalous) symmetries,

$$
\mathrm{SU}(N)_{\mathrm{c}} \times \mathrm{SU}(N+4) \times \mathrm{U}(1)_{\psi \eta} .
$$

It contains $N+2 \psi$ 's and $N+4 \eta$ 's, the correct numbers of the fermion zeromodes in the instanton background: in other words, it corresponds to a 't Hooft's instanton n-point function, e.g.,

$$
\left\langle\psi \eta \eta\left(x_{1}\right) \psi \eta \eta\left(x_{2}\right) \psi \eta\left(x_{3}\right) \ldots \psi \eta\left(x_{N+2}\right)\right\rangle .
$$

Up to now, we have assumed that the $\psi \eta$ system is in dynamical Higgs phase, characterized by the bifermion $\psi \eta$ condensate, (3.20). In fact, a crucial observation is that there is no way of saturating the fermion zero modes, $N_{\psi}=N+2 ; N_{\eta}=N+4$, by using the baryon fields $(B \sim \psi \eta \eta)$ only. This is a strong hint that such a confining phase in which the only infrared degrees of freedom are the baryons (appendix A) cannot be the correct vacuum of the system.

The problem of multi-valuedness of the effective action is solved by assuming that

$$
\left\langle\epsilon^{(4)} B B\right\rangle \neq 0, \quad\langle\operatorname{det} U\rangle \neq 0,
$$

where

$$
\epsilon^{(4)} B B=\epsilon_{\ell_{1} \ell_{2} \ell_{3} \ell_{4}} B^{\ell_{1} \ell_{2}} B^{\ell_{1} \ell_{2}}, \quad \ell_{i}=N+1, \ldots, N+4 .
$$

As

$$
\langle\operatorname{det} U\rangle \propto \mathbf{1}_{N \times N}
$$

takes up all flavors up to $N$ (by using the full $\mathrm{SU}(N+4)$ symmetry to orient the symmetry breaking direction), $B B$ must be made of the four remaining flavors, as in (3.27). These baryons were not considered in earlier studies [10, 25], but are assumed to be massless here, and indicated as $B^{\left[A_{2} B_{2}\right]}$ in table 2 (appendix B). Note that this appears to present us with another puzzle. If these extra baryons were massive, how could such composites appear in the low-energy effective action? Actually, there is an elegant answer. The point is that these extra baryons do not have conventional triangle anomalies with respect to the unbroken flavor symmetry,

$$
G_{\mathrm{f}}^{\prime}=\mathrm{SU}(N)_{\mathrm{cf}} \times \mathrm{SU}(4) \times \mathrm{U}(1)^{\prime}
$$

(in particular, they have a vanishing $\mathrm{U}(1)^{\prime}$ charge), as can be seen in table 2 (appendix B). We assume therefore that the baryons, indicated as $B^{[A, B]}$ in table 2 , are all massless, including those carrying the flavor indices $(A, B=N+1, \ldots, N+4)$. The conventional 't 
Hooft criterion with respect to $\mathrm{SU}(N)_{\mathrm{c}} \times \mathrm{SU}(4) \times \mathrm{U}(1)^{\prime}$ did not require them but does not exclude them either.

We are now ready to answer the questions raised at the end of section 2.3. We expand around the VEV,

$$
\begin{aligned}
\operatorname{det} U & =\langle\operatorname{det} U\rangle+\ldots \propto \mathbf{1}+\frac{i}{F_{\pi}^{(0)}} \phi_{0}+\ldots ; \\
\epsilon^{(4)} B B & =\left\langle\epsilon^{(4)} B B\right\rangle+\ldots \propto \mathbf{1}+\frac{i}{F_{\pi}^{(1)}} \phi_{1}+\ldots,
\end{aligned}
$$

where we appropriately re-normalized the fields, $\phi_{0}$ is the fluctuation of $\sum_{n}(\psi \eta)^{n n},(2.40)$, and $F_{\pi}^{(0)}$ and $F_{\pi}^{(1)}$ are some constants with dimension of mass.

Note that under the nonanomalous U(1) symmetries discussed in section 2.3,

$$
\left\langle\left[Q_{\psi \eta}, \epsilon B B \operatorname{det} U\right]\right\rangle=0, \quad\left\langle\left[Q_{f}, \epsilon B B \operatorname{det} U\right]\right\rangle=0
$$

as expected: the fluctuation of $\epsilon B B \operatorname{det} U$,

$$
\tilde{\phi} \equiv N_{\pi}\left[\frac{1}{F_{\pi}^{(0)}} \phi_{0}+\frac{1}{F_{\pi}^{(1)}} \phi_{1}\right], \quad N_{\pi}=\frac{F_{\pi}^{(0)} F_{\pi}^{(1)}}{\sqrt{\left(F_{\pi}^{(0)}\right)^{2}+\left(F_{\pi}^{(1)}\right)^{2}}},
$$

therefore does not represent the physical NG boson. Indeed, the strong-anomaly effective action (3.22) gives a quadratic mass term for $\tilde{\phi}$.

Vice versa, an orthogonal combination

$$
\phi \equiv N_{\pi}\left[\frac{1}{F_{\pi}^{(1)}} \phi_{0}-\frac{1}{F_{\pi}^{(0)}} \phi_{1}\right]
$$

does not get mass from (3.22). It is this field that represents (i.e., is the interpolating field of) the physical $\mathrm{U}(1)_{N G} \mathrm{NG}$ boson of the $\psi \eta$ model, not the naïve expectation $\phi_{0}$, discussed in section 2.3. The presence of two condensates, $\langle\operatorname{det} U\rangle$ and $\left\langle\epsilon^{(4)} B B\right\rangle$, is thus the key to answer the questions brought up at the end of section 2.3. In particular, any anomalous combination of $\mathrm{U}(1)_{\psi}$ and $\mathrm{U}(1)_{\eta}$ is spontaneously broken also, and $\mathrm{U}(1)_{a n}$ can indeed be regarded as a good analogue of the axial $\mathrm{U}(1)_{A}$ symmetry in QCD.

Let us check that everything fits together. We note that $\left\langle\epsilon^{(4)} B B\right\rangle \neq 0$ but $\langle B\rangle=0$. On the contrary, $\langle\operatorname{det} U\rangle \neq 0$ implies the condensates $\langle U\rangle \propto \mathbf{1}_{N \times N}$, as $U \sim \psi \eta$ is a scalar composite. Furthermore, the condensate $\left\langle\epsilon^{(4)} B B\right\rangle \neq 0$ is a singlet of the unbroken symmetry $G_{\mathrm{f}}{ }^{\prime}$ : it does not modify the symmetry breaking pattern in the Higgs phase of the $\psi \eta$ model, determined by the color-flavor locked bifermion condensate, $\langle\psi \eta\rangle \propto \mathbf{1},(3.10)$. Also, there are four-baryon couplings from (3.22), and due to Nambu-Jona-Lasinio mechanism, $B \sim B^{\left[A_{2} B_{2}\right]}$ acquires mass: they disappear from the massless spectrum, leaving the massless spectrum of the $\psi \eta$ model considered earlier [10, 25]. It might appear that this effectively brings us back to the simple-minded approach to the strong anomaly sketched at the beginning of this section, but as we saw above, a more careful treatment was really needed. 
The full understanding of the problem would require clarification of the mechanism for the condensation of the baryon pair together with that of $\operatorname{det} U,(3.26)$. The absolute values of the condensates $B B$ and $\operatorname{det} U=\operatorname{det}(\psi \eta)$ are flat directions of the potential (3.22). The instanton-induced potential is thus insufficient to determine them in itself. Even though the condensation of $\operatorname{det} U$ is analogous to the quark condensates in $\mathrm{QCD}$, and can be understood as due to the strong interactions among $\psi$ 's and $\eta$ 's with the color gauge bosons, the condensation of the color-singlet baryons is less obvious. Being the components of the baryon $B \sim \psi \eta \eta$ charged under color, it is natural to expect residual dipole-like interactions between the $B$ 's. The flat directions of the instanton potential (3.22) will be lifted, in some way, by quantum corrections. Such an information is implicitly in the (unkown) potential $V$ in (3.19), but certainly a more in-depth study (as in the Coleman-Weinberg effective action) is needed to understand the mechanism for the condensation of $B B$, and the relation with the $\operatorname{det} U$ condensate.

\subsection{Strong anomaly effective action in the generalized BY models}

As the solution given above on the $\psi \eta$ model is remarkably subtle, one might wonder whether a similar mechanism is at work in the so-called general Bars-Yankielowicz model, an $\mathrm{SU}(N)$ gauge theory with Weyl fermions

$$
\psi^{i j}, \quad \eta_{i}^{A}, \quad \xi^{i, a}
$$

in the direct-sum representation

$$
\square \oplus(N+4+p) \overleftarrow{\square} \oplus p \square .
$$

Without repeating the analysis we recall simply [25] that a chirally symmetric confining vacuum, with massless baryons

$$
\left(B_{1}\right)^{[A B]}=\psi^{i j} \eta_{i}^{A} \eta_{j}^{B}, \quad\left(B_{2}\right)_{A}^{a}=\bar{\psi}_{i j} \bar{\eta}_{A}^{i} \xi^{j, a}, \quad\left(B_{3}\right)_{\{a b\}}=\psi^{i j} \bar{\xi}_{i, a} \bar{\xi}_{j, b},
$$

(the first is anti-symmetric in $A \leftrightarrow B$ and the third is symmetric in $a \leftrightarrow b$ ), saturating all conventional 't Hooft anomaly triangles (see appendix E) cannot be the correct vacuum of the system. A $\left(\mathbb{Z}_{2}\right)_{F}-\left[\mathbb{Z}_{2}\right]^{2}$ mixed anomaly, present in the UV theory, is absent in the IR.

A dynamical Higgs phase (see appendix F) with condensate formation,

$$
\begin{aligned}
\left\langle U^{i B}\right\rangle & =\left\langle\psi^{i j} \eta_{i}^{B}\right\rangle=c_{\psi \eta} \Lambda^{3} \delta^{j B} \neq 0, \quad j, B=1, \ldots, N, \\
\left\langle V^{a A}\right\rangle & =\left\langle\xi^{i, a} \eta_{i}^{A}\right\rangle=c_{\eta \xi} \Lambda^{3} \delta^{N+4+a, A} \neq 0, \quad a=1, \ldots, p, \quad A=N+5, \ldots, N+4+p,
\end{aligned}
$$

and with symmetry breaking

$$
\begin{aligned}
\mathrm{SU}(N)_{\mathrm{c}} & \times \mathrm{SU}(N+4+p)_{\eta} \times \mathrm{SU}(p)_{\xi} \times \mathrm{U}(1)_{\psi \eta} \times \mathrm{U}(1)_{\psi \xi} \\
& \stackrel{\langle\xi \eta\rangle,\langle\psi \eta\rangle}{\longrightarrow} \mathrm{SU}(N)_{\mathrm{cf}_{\eta}} \times \mathrm{SU}(4)_{\eta} \times \mathrm{SU}(p)_{\eta \xi} \times \mathrm{U}(1)_{\psi \eta}^{\prime} \times \mathrm{U}(1)_{\psi \xi}^{\prime}
\end{aligned}
$$

turns out instead to be consistent [25]. 
A strong anomaly effective action for these theories can be constructed in a way analogous to the $\psi \eta$ model. Instead of (3.23), one has now

$$
\begin{aligned}
\epsilon & B_{1} B_{1} \operatorname{det} U \operatorname{det} V \\
\equiv & \epsilon^{m_{1}, m_{2}, \ldots, m_{N+4+p}} \epsilon^{i_{1}, i_{2}, \ldots, i_{N}} \epsilon^{k_{1}, k_{2}, \ldots, k_{p}} \\
& \times B_{1}^{\left[m_{N+1}, m_{N+2}\right]} B_{1}^{\left[m_{N+3}, m_{N+4}\right]} U^{i_{1} m_{1}} U^{i_{2} m_{2}} \ldots U^{i_{N} m_{N}} V^{m_{N+5} k_{1}} \ldots V^{m_{N+4+p} k_{p}},
\end{aligned}
$$

where $B_{1}$ are the baryons $\sim \psi \eta \eta$ defined in (3.36). The rest of the analysis closely follows that of the $\psi \eta$ model discussed in section 3.2. We shall not pursue further the details of the analysis here, except for noting that the strong anomaly effective action with such a logarithm, is perfectly consistent with (implies?) the condensates, (3.37), together with $\left\langle B_{1} B_{1}\right\rangle \neq 0$, where $B_{1}$ are the baryons defined in (3.36), (F.6). Writing extensively,

$$
\begin{aligned}
\left\langle B_{1} B_{1}\right\rangle & =\left\langle\epsilon_{C_{1} C_{2} C_{3} C_{4}}\left(\psi^{i j} \eta_{i}^{C_{1}} \eta_{j}^{C_{2}}\right)\left(\psi^{k \ell} \eta_{k}^{C_{3}} \eta_{\ell}^{C_{4}}\right)\right\rangle \neq 0, \\
C_{1} \sim C_{4} & =N+1, \ldots, N+4:
\end{aligned}
$$

i.e., the system is in dynamical Higgs phase, described in appendix F.

On the contrary, it is clearly not possible to write the strong-anomaly effective action with logarithmic argument (3.39), in terms of massless composite fermions (3.36).

\subsection{Strong anomaly in the $\chi \eta$ model}

Any combination of $\mathrm{U}(1)_{\chi}$ and $\mathrm{U}(1)_{\eta}$ other than $\mathrm{U}(1)_{\chi \eta}$ (see table 4 ) suffers from the strong anomaly. It means that the low-energy effective action should contain a term analogous to (3.4) for QCD or (3.12) for the $\psi \eta$ model. The natural choice of a gauge-invariant condensate (2.56) suggests an effective action of the form for the $\chi \eta$ model:

$$
\frac{i}{2} q(x) \log (\chi \eta)^{N-4} \chi \chi+\text { h.c. }
$$

$(q(x)$ is the topological density defined in (3.5)) where

$$
(\chi \eta)^{N-4} \chi \chi \equiv \epsilon_{i_{1} i_{2} \ldots i_{N}} \epsilon_{m_{1} m_{2} \ldots m_{N-4}}(\chi \eta)^{i_{1} m_{1}}(\chi \eta)^{i_{2} m_{2}} \ldots(\chi \eta)^{i_{N-4} m_{N-4}} \chi^{i_{N-3} i_{N-2}} \chi^{i_{N-1} i_{N}} .
$$

The argument of the logarithmic function taken here reflects the correct number of the fermion zeromodes in the instanton background $\left(N_{\chi}=N-2\right.$ and $\left.N_{\eta}=N-4\right)$; the epsilon tensors take care of the invariance under the full (nonanomalous) symmetry of the $\chi \eta$ system,

$$
\mathrm{SU}(N)_{\mathrm{c}} \times \mathrm{SU}(N-4) \times \mathrm{U}(1)_{\chi \eta} .
$$

This anomaly effective action agrees with the one proposed by Veneziano [5] for the special case of $\mathrm{SU}(5) \chi \eta$ model, and generalizes it to all $\mathrm{SU}(N) \chi \eta$ models.

An important observation we share with [5] is that this strong anomaly effective action, which should be present in the low-energy theory to reproduce correctly the (anomalous and nonanomalous) symmetries of the UV theory, implies nonvanishing condensates,

$$
\langle\chi \eta\rangle \neq 0, \quad\langle\chi \chi\rangle \neq 0,
$$

i.e., the dynamical Higgs phase, appendix D. 
Another important observation here is that there is no way of writing the strong anomaly effective action (3.41) in terms of the "baryons", $B \sim \chi \eta \eta$, of the assumed confining, chirally symmetric phase (appendix C). No combination of the baryons can saturate the correct number of the fermion zeromodes, $c f r(3.42)$.

Even though, contrary to the $\psi \eta$ model, the $\chi \eta$ system has no physical U(1) NG boson (it is eaten by a color $\mathrm{SU}(N)$ gauge boson), the counting of the broken and unbroken $\mathrm{U}(1)$ symmetries is basically similar in the two models. Of the two nonanomalous symmetries $\left(\mathrm{U}(1)_{\mathrm{c}}\right.$ and $\left.\mathrm{U}(1)_{\chi \eta}\right)$, a combination remains a manifest symmetry, and the other becomes the longitudinal part of the $T_{\mathrm{c}}$ gauge boson. Still another, anomalous, U(1) symmetry exists, any combination of $\mathrm{U}(1)_{\chi}$ and $\mathrm{U}(1)_{\eta}$ other than $\mathrm{U}(1)_{\chi \eta}$. This symmetry is also spontaneously broken hence must be associated with a NG boson, though it will get mass by the strong anomaly.

By expanding the composite $\chi \eta$ and $\chi \chi$ fields around their VEV's,

$$
\begin{aligned}
(\operatorname{det} U)^{\prime} & =\left\langle(\operatorname{det} U)^{\prime}\right\rangle+\ldots \propto \mathbf{1}+\frac{i}{F_{\pi}^{(0)}} \phi_{0}^{\prime}+\ldots, \\
\chi \chi & =\langle\chi \chi\rangle+\ldots \propto \mathbf{1}+\frac{i}{F_{\pi}^{(1)}} \phi_{1}^{\prime}+\ldots,
\end{aligned}
$$

where $(\operatorname{det} U)^{\prime}$ is defined in the $N-4$ dimensional color-flavor mixed space, and

$$
\chi \chi=\epsilon_{i_{1} i_{2} i_{3} i_{4}} \chi^{i_{1} i_{2}} \chi^{i_{3} i_{4}}, \quad N-3 \leq i_{j} \leq N .
$$

Now the strong-anomaly effective action (3.41) gives mass to

$$
\tilde{\phi}^{\prime} \equiv N_{\pi}\left[\frac{1}{F_{\pi}^{(0)}} \phi_{0}^{\prime}+\frac{1}{F_{\pi}^{(1)}} \phi_{1}^{\prime}\right], \quad N_{\pi}=\frac{F_{\pi}^{(0)} F_{\pi}^{(1)}}{\sqrt{\left(F_{\pi}^{(0)}\right)^{2}+\left(F_{\pi}^{(1)}\right)^{2}}},
$$

whereas an orthogonal combination

$$
\phi^{\prime} \equiv N_{\pi}\left[\frac{1}{F_{\pi}^{(1)}} \phi_{0}^{\prime}-\frac{1}{F_{\pi}^{(0)}} \phi_{1}^{\prime}\right]
$$

remains massless: it is this potential NG boson which is absorbed by the color $T_{\mathrm{c}}$ gauge boson.

\subsection{Strong anomaly in the generalized GG models}

The structure of the strong anomaly action in the $\chi \eta$ turned out to be markedly simpler than that in the $\psi \eta$ model. The argument of the logarithm is made of composite scalar fields only. One might wonder if such a simple description is valid also for more general Georgi Glashow models [25], an $\mathrm{SU}(N)$ gauge theory with Weyl fermions

$$
\chi^{[i j]}, \quad \eta_{i}^{A}, \quad \xi^{i, a}
$$

in the direct-sum representation

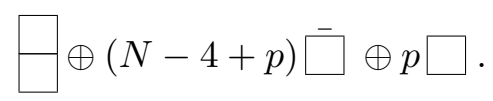


It turns out that the construction (3.41) straightforwardly generalizes to

$$
\frac{i}{2} q(x) \log \epsilon \chi \chi \operatorname{det}(\chi \eta) \operatorname{det}(\xi \eta)+\text { h.c. , }
$$

where we used a shorthand notation

$$
\begin{aligned}
& \epsilon \chi \chi \operatorname{det}(\chi \eta) \operatorname{det}(\xi \eta) \\
& =\epsilon_{i_{1} i_{2} \ldots i_{N}} \epsilon_{k_{1} k_{2} \ldots k_{p}} \epsilon_{m_{1} m_{2} \ldots m_{N-4+p}} \\
& \quad \times(\chi \eta)^{i_{1} m_{1}}(\chi \eta)^{i_{2} m_{2}} \ldots(\chi \eta)^{i_{N-4} m_{N-4}} \chi^{i_{N-3} i_{N-2}} \chi^{i_{N-1} i_{N}}(\xi \eta)^{m_{N-3} k_{1}} \ldots(\xi \eta)^{m_{N-4+p} k_{p}} .
\end{aligned}
$$

Note that this is a singlet of the full symmetry group of the model. The strong anomaly action (3.51) implies the condensates

$$
\begin{aligned}
\left\langle\chi^{i j} \eta_{i}^{A}\right\rangle & =c_{\chi \eta} \Lambda^{3} \delta^{j A} \neq 0, & & j=1, \ldots, N-4, \quad A=1, \ldots, N-4, \\
\left\langle\xi^{i, a} \eta_{i}^{B}\right\rangle & =c_{\xi \eta} \Lambda^{3} \delta^{N-4+a, B} \neq 0, & & a=1, \ldots, p, \quad B=N-3, \ldots, N-4+p,
\end{aligned}
$$

and

$$
\left\langle\chi^{j_{1} j_{2}} \chi^{j_{3} j_{4}}\right\rangle=c_{\chi \chi} \epsilon^{j_{1} j_{2} j_{3} j_{4}} \Lambda^{3} \neq 0, \quad j_{1}, \ldots, j_{4}=N-3, \ldots, N
$$

These are precisely the set of condensates expected to occur in the Higgs phase of the GG models, appendix $\mathrm{H}$ [25].

Vice versa, in the confining vacuum with unbroken global symmetry, appendix G, there is no way the baryons (G.2) saturate all the fermion zeromodes, as in (3.52).

\section{6 $\mathrm{SU}(6)$ model with a single fermion in a self-adjointrepresentation}

After the studies of BY and GG models above, it may be of some interest to see whether our argument based on the strong anomaly may give some relevant information in other types of models. Let us discuss just a few examples. The first is an SU(6) model with a single left-handed fermion in the representation,

$$
\underline{20}=\square,
$$

studied in $[33,34]$. The gauging of the 1 -form $\mathbb{Z}_{3}^{C}$ symmetry gives rise to a new anomaly of the nonanomalous $\mathbb{Z}_{6}^{\psi}$ symmetry,

$$
\mathbb{Z}_{6}^{\psi} \longrightarrow \mathbb{Z}_{2}^{\psi}
$$

leading to a three-fold vacuum degeneracy [33, 34]. The interpretation and the details of such a breaking depends on which kind of condensates are formed in the infrared. As (in this particular model), a scalar bifermion composite cannot be a gauge singlet, the author of [33] suggested a four-fermion condensate

$$
\langle\psi \psi \psi \psi\rangle \sim \Lambda^{6} \neq 0, \quad\langle\psi \psi\rangle=0
$$


whereas the authors of [34] proposed the gauge symmetry breaking condensate

$$
\langle\psi \psi\rangle \sim \Lambda^{3} \neq 0
$$

with $\psi \psi$ in the adjoint representation of $\mathrm{SU}(6)$. Both scenarios turn out to be consistent with the discrete symmetry breaking, (3.56), and hence agree on a three-fold vacuum degeneracy, but the infrared physics associated with these assumptions are quite different, and one wonders which of the assumptions describes the system in the infrared.

The strong anomaly effective action in this model has the form,

$$
\frac{i}{2} q(x) \log \psi \psi \psi \psi \psi \psi+\text { h.c. . }
$$

Requiring that the argument of the log acquires a nonvanishing VEV, the assumption of four-fermion condensate (3.57) appears to be somewhat unnatural, whereas the bifermion condensates (3.58) looks perfectly consistent, with

$$
\left\langle\psi \psi \psi \psi \psi \psi \psi \sim\langle\psi \psi\rangle_{j}^{i}\langle\psi \psi\rangle_{k}^{j}\langle\psi \psi\rangle_{i}^{k} \neq 0,\right.
$$

where the color indices are briefly restored.

\subsection{Adjoint QCD with $N_{\mathrm{c}}=N_{\mathrm{f}}=2$}

Another interesting model is the adjoint QCD, widely studied in the literature. Let us however consider a particular case, $N_{\mathrm{c}}=N_{\mathrm{f}}=2$. The fermions are two Weyl fermions $\lambda^{i}$, $i=1,2$, both in the adjoint of $\mathrm{SU}(2)$. The conventional thinking assumed that a gauge invariant bifermion condensate

$$
\langle\lambda \lambda\rangle \neq 0
$$

forms, breaking the flavor symmetry as $\mathrm{SU}(2)_{\mathrm{f}} \rightarrow \mathrm{SO}(2)_{\mathrm{f}}$, leading to $2 \mathrm{NG}$ bosons, and reducing the discrete $\mathbb{Z}_{8}$ symmetry to $\mathbb{Z}_{2}$ resulting four degenerate vacua.

A special interest in this model was raised by the work by Anber and Poppitz [35], which postulates that the system develops a condensates,

$$
\langle\lambda \lambda \lambda \lambda\rangle \neq 0, \quad\langle\lambda \lambda\rangle=0,
$$

breaking the discrete symmetry as $\mathbb{Z}_{8}$ symmetry to $\mathbb{Z}_{4}$, hence predicting a doubly degenerate vacua. The flavor $\mathrm{SU}(2)_{\mathrm{f}}$ remains unbroken; massless baryons

$$
\sim \lambda \lambda \lambda
$$

(which is necessarily a doublet of $\mathrm{SU}(2)_{\mathrm{f}}$ ) saturates Witten's $\mathrm{SU}(2)$ anomaly.

The generalized, mixed anomaly study in this model predicts [34, 35] the discrete symmetry breaking $\mathbb{Z}_{8} \rightarrow \mathbb{Z}_{4}$ : such a result is consistent with both of the dynamical scenarios mentioned above, which are markedly different from physics point of view.

Does our strong-anomaly argument tell anything significant? The analogue of the strong anomaly effective action, such a (3.4, (3.41) and (3.59), is in this case,

$$
\frac{i}{2} q(x) \log \lambda \lambda \ldots \lambda+\text { h.c. },
$$


with eight $\lambda$ 's inside the argument of the logarithmic function. In this model, in contrast to what we saw in the preceding model, section 3.6, our algorithm seems to be consistent with either of the dynamical possibilities, (3.61), or (3.62).

It would be interesting if a lattice study could give a definitive answer.

As a side comment, in the case with $N_{\mathrm{f}}=1$, arbitrary $N_{\mathrm{c}}$, the adjoint QCD becomes $\mathcal{N}=1$ supersymmetric Yang-Mills, and (3.64) with $2 N_{\mathrm{f}} N_{\mathrm{c}}=2 N_{\mathrm{c}} \quad \lambda$ 's, reduces precisely to the Veneziano-Yankielowicz [27] effective potential. And in that case, the assumption of the bifermion condensate, $\langle\lambda \lambda\rangle \neq 0$, the breaking of the discrete symmetry $\mathbb{Z}_{2 N_{\mathrm{c}}} \rightarrow \mathbb{Z}_{2}$, and the resulting $N_{\mathrm{c}}$ fold degeneracy of the vacua (equal to Witten's index), are by now generally accepted as the correct answer for these systems. ${ }^{7}$

\section{Complementarity in the $\chi \eta$ model?}

In the $\chi \eta$ model the symmetry of the massless sector in the dynamical Higgs phase (2.43) (appendix D) happens to coincide ${ }^{8}$ with that of the UV theory hence with that of the symmetric, confining phase (appendix C). This fact might lead some, based on the socalled complementarity picture [40], to think that in the $\chi \eta$ model these two phases are actually one and the same.

The following discussion however shows that the coincidence of the global symmetries in the two candidate vacua in the $\chi \eta$ model is an accidental one. There are, indeed, clear indications that they are physically distinct [10]. Of course it would be difficult to think that a phase in which

$$
\langle\chi \eta\rangle \propto \Lambda^{3} \neq 0, \quad\langle\chi \chi\rangle \propto \Lambda^{\prime 3} \neq 0
$$

(with no adjustable constants in front, and $\Lambda \sim \Lambda^{\prime}$ is the dynamical mass scale generated by the strong $\mathrm{SU}(N)$ gauge interactions.), and another, without any condensates, can be the same phase. There are no coupling constants which can be varied continuously so that the two possible "phases" (one with (4.1), one with no condensates) are connected without phase transition.

Another relevant issue could be the fact that in the dynamical Higgs phase (appendix D) there appear $(N-4)^{2}-1$ massive gauge bosons of degenerate mass, by the Higgs mechanism together with the remnant color-flavor diagonal $\mathrm{SU}(N-4)$ global symmetry. This is a clear prediction which can be tested by lattice simulations. Even though it is true that these massive bosons can be re-expressed as gauge-invariant composites, there is no particular reason why precisely these massive bosons and not others should appear at the mass scale $\sim \Lambda$, if the system is in confining, symmetric phase of appendix $\mathrm{C}$.

Also, to understand the infrared dynamics, it is indispensable to take into account of the effects of strong anomaly correctly. Its natural form is (3.41), as discussed in the

\footnotetext{
${ }^{7}$ Lattice studies [36-38] also seem to confirm this. We thank Stefano Piemonte for bringing these references to our attention.

${ }^{8}$ As noted in [25] this occurs only for the $\chi \eta$ model. In all other generalized Bars-Yankielowicz and Georgi-Glasow models, the dynamical Higgs phase has global symmetry distinct from that in the confining, no-condensate phase, in spite of the fact that one of the bifermion condensate is in the fundamental representation of the gauge group. See appendices A-H.
} 
previous section, by taking into account the fermion zeromodes in an instanton background. The solution of the effective equation of motion would then lead to a massive NG boson, but as in QCD and in the $\psi \eta$ model, the very solution implies nonvanishing vacuum condensate, (4.1). It means that the system is in dynamical Higgs phase.

Crucially, as already noted at the end of section 3.4, there is no way of writing the strong-anomaly effective action in terms of the massless composite "baryons" $(\sim \chi \eta \eta)$ : the zero-mode counting $\left(N_{\chi}=N-2>N_{\eta}=N-4\right)$ cannot work. This is a clear sign that the "confining phase" (Appndix C) can neither be equivalent to the Higgs phase (appendix D) nor is one of consistent vacuum phases of the $\chi \eta$ model.

Of course, one of the strongest arguments which discriminates the two candidate vacua comes from the mixed anomalies and the consequent, generalized-anomaly-matching analysis $[25,26]$ (see also [39]). The presence of a mixed anomaly of the type $\left(\mathbb{Z}_{2}\right)_{F}-\left[\mathbb{Z}_{N}\right]^{2}$ in the UV theory and the absence of this anomaly in the "symmetric confining phase", show that such a phase cannot be the correct vacuum of the theory [25, 26]. As in the $\psi \eta$ model, in the dynamical Higgs phase characterized by the bifermion condensates (4.1), the color-flavor locked $\mathbb{Z}_{N}$ symmetry is spontaneousy broken by the condensates, as the $\mathrm{U}(1)_{\chi \eta}$ symmetry used to form $\mathbb{Z}_{N} \subset \mathrm{U}(1)_{\chi \eta} \times\left(\mathbb{Z}_{2}\right)_{F}$ is spontaneously broken.

To summarize: both the mixed-anomaly analysis $[25,26]$ and the consideration of the strong-anomaly effective action section 3.4, lead to the same conclusion that one of the candidate phase (dynamical Higgs phase) is consistent whereas the other (confining, flavor symmetric phase) is inconsistent, but not that these two phases are inequivalent, perhaps separated by a phase transition of some sort.

\section{Large $N$ planar dominance and U(1) NG bosons in chiral gauge the- ories}

Large $N$ counting and planar Feynman diagram dominance are perturbative concepts and are not adequate for describing condensates. Nevertheless, an argument was presented some time ago [7] which states that in some chiral gauge theories such as $\psi \eta$ or $\chi \eta$ models, a U(1) symmetry cannot be spontaneously broken, if the system is assumed to confine. Such a statement, if taken without due care, might mislead the reader to conclude that, e.g., in the $\psi \eta$ model, the only possible phase is the chirally symmetric phase, with no condensates and with massless composite fermions (appendix A). ${ }^{9}$

To be scrupulous, the authors of [7] do not claim that a dynamical Higgs phase (with gauge dependent bifermion condensate) is impossible in the $\psi \eta$ model, or in general chiral gauge theories. Indeed one of the two main conclusions of [7] is that a dynamical Higgs phase is likely to be the correct answer for some chiral gauge theories (e.g., the model in section 5 of [7]). Their claim concerning the $\psi \eta$ model (section 3 of [7]) is that if the $\psi \eta$ system is in an exact confinement phase, then a two point function of a broken $\mathrm{U}(1)$ current $\left\langle 0\left|T\left\{J_{\mu}(x) J_{\nu}(0)\right\}\right| 0\right\rangle$ cannot have, in the leading $N$ approximation, an intermediate state having the quantum numbers of the $\mathrm{U}(1) N G$ boson, therefore a spontaneous $\mathrm{U}(1)$ breaking is not possible.

\footnotetext{
${ }^{9}$ Let us remember that the notation in [7] and in the present work are simply related by $S=\psi ; \bar{F}=\eta$.
} 
Nevertheless, there seem to be a number of issues in this argument. First of all, no definition of "exact confinement" is given in [7]. We noted already that there is no global center symmetry which can be used with the Polyakov loop, to set a criterion for discriminating confinement/Higgs phases. The authors of [7] describe the "confinement phase" as a phase in which all observable states are gauge invariant, but this is also problematic, as in a local gauge theory gauge noninvariant (gauge-dependent) states can always be re-expressed in a (though more complicated) gauge-invariant representation. See (2.40) versus (2.41), for instance.

If the distinction ("exact confinement" or not) is whether or not a gauge-dependent condensate forms, this cannot be used as a criterion either: as shown in section 2.3, the gauge-dependent $\psi \eta$ condensate can be re-expressed as a gauge invariant multifermion condensate, (2.41).

If "exact confinement" means, in the context of the $\psi \eta$ model, that no condensates $\langle\psi \eta\rangle \neq 0$ form, then no $\mathrm{U}(1)$ symmetry is broken spontaneously, hence no NG bosons would appear in the system anyway, independently of the $\frac{1}{N}$ counting. The observation [7] that in the large $N$ limit, assuming the planar diagram dominance, the intermediate states are all made of pairs of outgoing and incoming $\psi$ 's and $\eta$ 's, hence are all neutral with respect to the $\mathrm{U}(1)$ charge, is not exactly pertinent in this case.

Vice versa, if the condensate $\langle\psi \eta\rangle$ does occur, and thus the system is in Higgs phase, then there is a $\mathrm{U}(1)$ symmetry which is spontaneously broken, and a physical NG boson appears (see section 2.3 above). This NG boson can appear as an intermediate state of the two-point function of the associated currents, in the leading $N$ planar graph.

Indeed, for the large $N$ counting of Feynman diagrams in the $\psi \eta$ model, it is sufficient to remember [7] that neither $\psi$ nor $\eta$ loops are suppressed by $\frac{1}{N}$ : the standard $\frac{1}{N}$ suppression for the fundamental-fermion $(\eta)$ loop inside a planar graph is compensated by a factor $\sim N$ from the flavor multiplicity. This leads to the observation [7] that the diagrams with $B \bar{B}$, $B \bar{B} B \bar{B}$, etc., intermediate states $^{10}$ are not suppressed by a $\frac{1}{N}$ factor.

For exactly the same reason, neither are the planar graphs with the intermediate states $\eta \psi \psi^{*} \eta^{*}, \eta \psi \eta \psi(\psi \eta)^{*}(\psi \eta)^{*}$, and $\operatorname{det} U(\operatorname{det} U)^{*}{ }^{11} \mathrm{~A}$ simplest such graph is shown in figure 1. Allowing for nonvanishing condensates $\langle\psi \eta\rangle$ to occur in the diagram, one can see that the U(1) NG boson does appear as an intermediate state in the current two-point function, figure 2. There is no difficulty showing the same by using the gauge-invariant condensate and pion field, (2.41), even though it becomes more cumbersome to draw a picture.

Recapitulating, a large $N$ counting argument neither discriminates the possible phases nor prohibits a U(1) symmetry to be broken spontaneously.

\section{Multifield versus bi-fermion condensates}

One of the key elements of our argument based on the strong anomaly is the statement that the multifield condensate such as the one in the $\chi \eta$ model,

$$
\left\langle(\chi \eta)^{N-4} \chi \chi\right\rangle \neq 0
$$

\footnotetext{
${ }^{10} B$ are the hypothetical massless composite fermions ("baryons") of (A.1), $B \sim \psi \eta \eta$.

${ }^{11}$ The $\psi$ and $\eta$ loops (rings) in the graph must be ordered one inside the other, alternatively, in order to keep the leading $\frac{1}{N}$ order. Arbitrary gluon exchanges can be added in a planar graph as in figure 1 .
} 

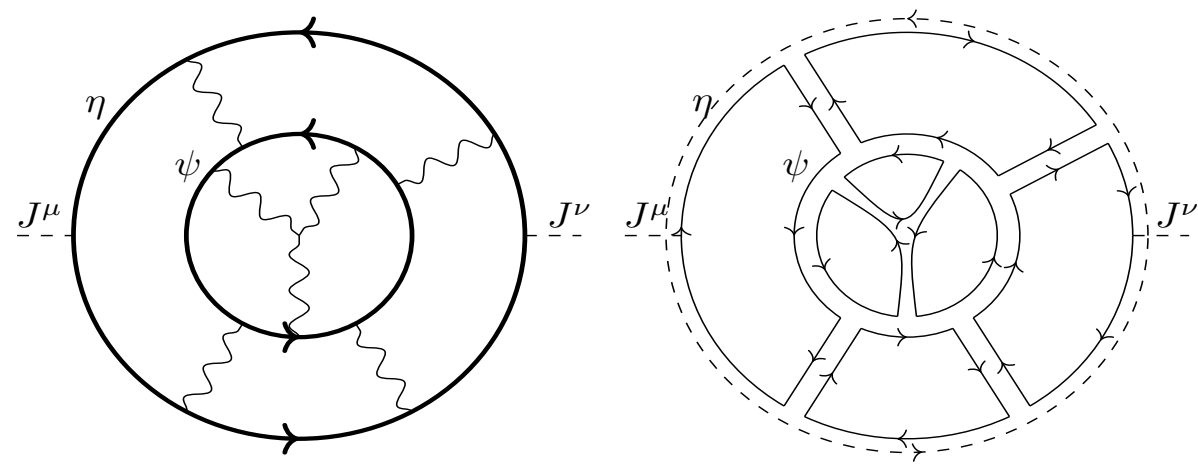

Figure 1. A leading $N$ planar graph for the two-point function $\left\langle 0\left|T\left\{J_{\mu}(x) J_{\nu}(0)\right\}\right| 0\right\rangle$ with an $\eta^{*} \psi^{*} \psi \eta$ intermediate state (figure 1a, left). On the right figure (figure 1b) the color (full) and flavor (dashed) lines are shown. The wavy lines inside the graph are the gluons.

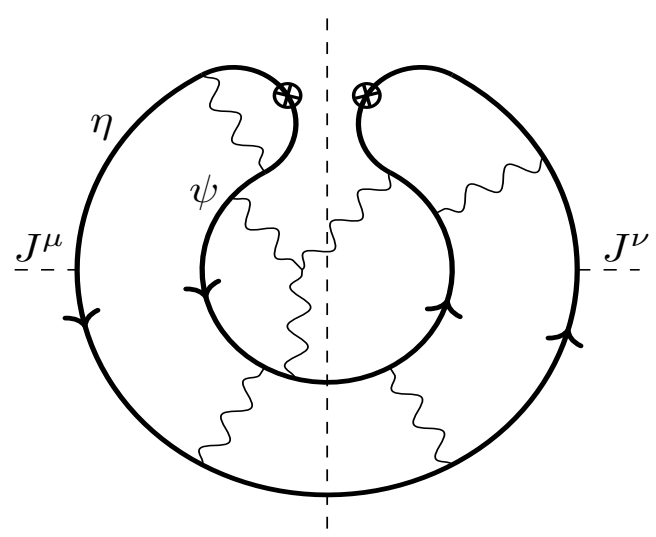

Figure 2. The same graph as figure 1, with insertion of $\langle\psi \eta\rangle$ condensates (shown with $\otimes$ ). The intermediate state now corresponds to the $\mathrm{U}(1) \mathrm{NG}$ boson $\sim \psi \eta$ of eq. (2.40).

(see eq. (3.42) for the exact meaning) implies the condensation of the component bifermion scalars,

$$
\langle\chi \eta\rangle \neq 0, \quad\langle\chi \chi\rangle \neq 0
$$

where

$$
\begin{array}{ll}
\langle\chi \eta\rangle=\left\langle\chi_{[i j]} \eta^{B j}\right\rangle \sim \Lambda^{3} \delta_{i}^{B}, & i, B=1,2, \ldots, N-4, \\
\langle\chi \chi\rangle=\left\langle\epsilon_{i_{1} i_{2} i_{3} i_{4}} \chi^{i_{1} i_{2}} \chi^{i_{3} i_{4}}\right\rangle \sim \Lambda^{3}, & N-3 \leq i_{j} \leq N,
\end{array}
$$

in an appropriate gauge and with flavor orientation. A similar step is used in all other models considered here. As this point is central in our discussion, let us pose, before summarizing and concluding this paper, to make a few more comments. We will use the $\chi \eta$ model (the simplest of the GG models) for this purpose, for concreteness and to keep the notations simple.

We have not proven that (6.2) follows from (6.1). We do believe this is correct, but our deduction is based on a collection of plausibility considerations and consistency checks: 
dealing with a strongly-coupled gauge theories, to mathematically prove this type of derivation is not always an easy task.

Here are a few more considerations, supporting our deduction.

(a) Quark condensates in $Q C D$ versus $\chi \eta$ condensate: in $\mathrm{QCD}$, the quark condensate

$$
\left\langle\bar{\psi}_{R} \psi_{L}\right\rangle \sim \Lambda^{3} \neq 0
$$

does not imply

$$
\left\langle\bar{\psi}_{R}\right\rangle \neq 0, \quad\left\langle\psi_{L}\right\rangle \neq 0
$$

However this is not because the quarks are not gauge invariant, but because they are fermions.

But then as we recalled in section 3.1, the multifield condensate

$$
\langle\operatorname{det} U\rangle=\left\langle\operatorname{det} \bar{\psi}_{R} \psi_{L}\right\rangle \neq 0
$$

following from the strong-anomaly effective action, does seem to imply the bi-fermion scalar condensates, $\left\langle\bar{\psi}_{R} \psi_{L}\right\rangle \neq 0$. We will further argue in section 7 that the scalar composites $\bar{\psi}_{R} \psi_{L}$ in QCD and $\chi \eta$ in the GG model (and similarly $\psi \eta$ in the BY model, etc.) should be regarded as analogous objects.

We may further add that if the $\mathrm{SU}(2)_{W S} \times \mathrm{U}(1)_{Y}$ gauge interactions are taken into account, the quark condensates $\left\langle\bar{\psi}_{R} \psi_{L}\right\rangle \neq 0$ are no longer gauge-invariant objects. ${ }^{12}$ In this sense, too, there is no qualitative difference between the scalar composites $\bar{\psi}_{R} \psi_{L}$ in QCD and $\chi \eta$ in the GG model.

(b) The standard-model Higgs doublet: in the Weinberg-Salam model, the vacuum is at the gauge-invariant minimum of the potential,

$$
\left\langle\sum_{i=1}^{2} \phi^{i *} \phi^{i}\right\rangle \neq 0
$$

This is taken to mean a nonvanishing Higgs VEV, in an appropriate gauge,

$$
\langle\phi\rangle=\left(\begin{array}{l}
v \\
0
\end{array}\right), \quad v \neq 0 .
$$

Our deduction $(6.1) \leftrightarrow(6.2)$ is analogous to this, even though here we have a dynamical, composite "Higgs" scalars. One can argue against the use of this analogy, on the basis that the Weinberg-Salam model is a weakly coupled theory, while here we have a strongly coupled one. We believe that this difference (the former described

\footnotetext{
${ }^{12}$ As is well known, the quark condensate breaks the Weinberg-Salam gauge symmetry dynamically, even though only by a tiny amount, insufficient to explain in itself the observed electroweak gauge symmetry breaking.
} 
by a potential, the latter not) is not essential. What excludes the conclusion that the Weinberg-Salam model with (6.8) is in confinement phase with

$$
\left\langle\sum_{1}^{2} \phi^{i *} \phi^{i}\right\rangle \neq 0, \quad\langle\phi\rangle=0
$$

is the mass spectrum $(W, Z, \gamma$, etc). The attempt to rewrite the whole WeinbergSalam model as a confining theory [41] fails to reproduce exactly the mass spectra (though it does almost) of the standard Higgs phase description.

Our observation here is similar: even though the global symmetry may look the same in two "complementary" descriptions of the $\chi \eta$ model,${ }^{13}$ the exact mass spectra are probably different, as we pointed out in section 4 .

(c) Consistency: a more indirect, consistency argument is the following. Let us assume the multifield condensate (6.1) forms but with

$$
\langle\chi \eta\rangle=\langle\chi \chi\rangle=0
$$

and with the full (nonanomalous) symmetry of the $\chi \eta$ system,

$$
\mathrm{SU}(N)_{\mathrm{c}} \times \mathrm{SU}(N-4) \times \mathrm{U}(1)_{\chi \eta}
$$

intact. The low-energy system is then described, as the conventional 't Hooft anomaly argument suggests, by the set of massless baryons (C.1). But as there is no way to describe the mutifermion condensate (6.1) in terms of these massless fields, we conclude that the assumption (6.11) is inconsistent.

One may ask why the confining phase with baryons cannot have some composite scalar $\sim(\chi \eta)^{N-4} \chi \chi$ entering strong anomaly effective action. To answer this, we recall our criterion: the infrared degrees of freedom of the assumed phase must be able to describe the strong anomaly effectively at low energies. Just as the NG boson fields do in the Higgs phase of the GG models, and as they do in the confining phase in QCD. In the BY models, the massless baryons and NG bosons together saturate the argument of the logarithm, in the dynamical Higgs phase, as we discussed in section 3 .

If one adopts this criterion, then the confining, symmetric phase is disfavored (inconsistent) in all of the GG and BY models.

\section{Summary and discussion}

Consideration of the strong-anomalies, not always taken into account in the study of dynamics of chiral gauge theories, turns out to provides us with a powerful new argument

\footnotetext{
${ }^{13}$ Remember that the "complementarity" aspect is specific to the $\chi \eta$ model, not shared by any other GG or BY models.
} 
supporting the conclusion of $[25,26]$ : the system is in dynamical Higgs phase in the BarsYankielowicz and Georgi-Glashow models. The essential requirement we make is that it should be possible to write a strong-anomaly effective action by utilizing only the massless degrees of freedom, assumed to be present in the infrared. This simple requirement is naturally satisfied in all models considered, if assumed to be in the dynamical Higgs vacuum with bifermion condensates. On the contrary, in the putative, confinement vacua with massless composite fermions (baryons) as the only infrared degrees of freedom, we found no way of writing the correct strong-anomaly effective action. In other words, it seems that in these latter vacua, the anomalous U(1) symmetry(ies) cannot be properly realized in the infrared.

The fact that the matter fermions in our models have larger multiplicities than the quarks in QCD (for instance $\eta$ in the $\psi \eta$ model has the flavor multiplicity, $N+4 \sim N$ at large $N$ ), may mean that the mass of the anomalous $\mathrm{U}(1) \mathrm{NG}$ bosons will turn out to be of the oder of $\Lambda$. Even though we do write the strong-anomaly effective action in terms of the massless degrees of freedom of the presumed infrared theory, the fact that its solution (the minimum of the effective potential) is at $\mu \sim \Lambda$, means that the answer cannot be regarded as reliable quantitatively. We are however interested here only in a qualitative question of the validity of confinement vs dynamical Higgs phase in the infrared, and we do believe that our discussion of section 3 makes reasonable sense.

The fact that both mixed anomalies $[25,26]$ and the strong-anomaly effective action (this paper) imply dynamical Higgs phase in chiral BY and GG models - is actually not a pure coincidence. Both indeed arise by taking properly the strong chiral U(1) anomalies into account.

Certain analogies and contrasts between the strong-interaction dynamics of vectorlike and chiral gauge theories seem to emerge from these discussions. As the "confining, flavor symmetric" vacua have been shown to be disfavored, we assume below that these chiral gauge theories are indeed in dynamical Higgs phase.

And let us compare the physics of the $\psi \eta, \chi \eta$ and of more general BY and GG models, with that of the standard QCD with $N_{\mathrm{f}}$ light flavors of quarks and antiquarks. Both in QCD and in these chiral gauge theories, the theory without the matter fermions is the same, pure $\mathrm{SU}(N)$ Yang-Mills theory. We have here nothing new to add to the known and unknown properties of the pure $\mathrm{SU}(N)$ dynamics. Our interest here is the role the light matter fermions play in determining the infrared dynamics, and how the resulting phase(s) depend on the types of the matter fermions present.

In many senses, the bifermion condensates such as $U=\psi \eta$ in the $\psi \eta$ model (and $U=\chi \eta$ and $\chi \chi$ condensates in the $\chi \eta$ model), are a good analogue of the quark condensate $U=\bar{\psi}_{R} \psi_{L}$ in QCD. This "analogy" is based on the fact that all of these composite scalar fields enter the strong-anomaly effective action in the same way, as

$$
\hat{L}=\frac{i}{2} q(x) \log \operatorname{det} U / U^{\dagger}, \quad q(x)=\frac{g^{2}}{32 \pi^{2}} F_{\mu \nu}^{a} \tilde{F}^{a, \mu \nu},
$$

(see section 3 for more careful discussions). And in all cases this implies condensation, $\langle U\rangle \propto \mathbf{1}$, although it's meaning depends on the system: the color-flavor locked Higgs phase in the chiral models, and the chiral-symmetry-breaking confining vacuum in QCD. 
Also, in a more general Bars-Yankielowicz models, there are two natural bifermion condensation channels $((\cdot)$ meaning an $\mathrm{SU}(N)$ singlet $)$ :

$$
\begin{array}{rlr}
\psi(\square) \eta(\square) & \text { forming } & \square, \\
\xi(\square) \eta(\square) & \text { forming } & (\cdot):
\end{array}
$$

the gluon-exchange strengths in the two channels are, respectively, proportional to $-\frac{(N+2)(N-1)}{N}$ and $-\frac{N^{2}-1}{N}$. The $\psi \eta$ channel is slightly more attractive, but the strengths are identical in the large $N$ limit. Note that in our notation $\langle\xi \eta\rangle$ has the same quantum numbers as $\left\langle\bar{\psi}_{R} \psi_{L}\right\rangle$ in QCD. Similarly for the comparison between the condensates $\langle\chi \eta\rangle$ and $\langle\xi \eta\rangle$ in the general Georgi-Glashow models. These considerations, which are based on rather naïve MAC [2] like idea and therefore are not very rigorous, nevertheless show that the quark condensates in QCD and the bifermions condensates in the chiral gauge theories under study, are really on a very "similar" footing.

Of course, the fact that the quark condensate $\left\langle\bar{\psi}_{R} \psi_{L}\right\rangle$ is a color singlet, $\mathrm{SU}\left(N_{\mathrm{f}}\right)_{L} \times$ $\mathrm{SU}\left(N_{\mathrm{f}}\right)_{R}$ flavor matrix, whereas $\langle\psi \eta\rangle$ is in a color-flavor bifundamental form, breaking the color completely, makes a world of differences in other aspects of these (vectorlike or chiral) systems. Most importantly, the existence of colored NG bosons in the $\psi \eta$ (or in the $\chi \eta$ ) model, see section 2.2.1, means that these are coupled linearly to the color gauge bosons, and via Englert-Brout-Higgs mechanism make them massive. These processes are absent in QCD, all NG bosons being color singlets. It is in this sense that we talk about confinement phase in QCD, in spite of the fact that the linear potential between any two test particles can be always flattened by the pair production of quarks from the vacuum, and Higgs phase in the chiral models.

Also, the mass spectra get arranged quite differently in QCD and in the chiral models discussed here. Apart from the pattern of certain degenerate massive vector bosons (see section 4), the massless spectrum exhibits striking differences. In all chiral gauge theories considered here, it contains in general both a number of composite fermions (baryons) as well as some composite scalars (pions), a feature not shared by massless QCD. In other words, the way the chiral symmetries of the UV theory are realized in the IR, is distinctly different.

Perhaps a closer analogy - from a formal point of view - between the vectorlike theories and chiral theories, comes from the consideration of color superconductivity in the high-density limit of QCD [42, 43]. In such a situation the dynamics of QCD is believed to be such that the colored di-quark condensates form,

$$
\left\langle\psi_{L} \psi_{L}\right\rangle \neq 0, \quad\left\langle\psi_{R} \psi_{R}\right\rangle \neq 0
$$

In particular, for $N_{\mathrm{f}}=3$, they are condensates of color-flavor diagonal forms, somewhat similar to $\langle\psi \eta\rangle$ or $\langle\chi \eta\rangle$ in the chiral theories discussed here, although the details of the dynamics can obviously be quite different.

What lessons should one draw from these discussions? Clearly, there are both similarities and differences between the dynamics of QCD and that of the chiral gauge theories 


\begin{tabular}{|c|c|c|c|c|}
\hline & fields & $\mathrm{SU}(N)_{\mathrm{c}}$ & $\mathrm{SU}(N+4)$ & $\mathrm{U}(1)_{\psi \eta}$ \\
\hline \multirow{2}{*}{$\mathrm{UV}$} & $\psi$ & $\square$ & $\frac{N(N+1)}{2} \cdot(\cdot)$ & $N+4$ \\
& $\eta^{A}$ & $(N+4) \cdot \bar{\square}$ & $N \cdot \square$ & $-(N+2)$ \\
\hline IR & $B^{[A B]}$ & $\frac{(N+4)(N+3)}{2} \cdot(\cdot)$ & $\boxminus$ & $-N$ \\
\hline
\end{tabular}

Table 1. Chirally symmetric "confining" phase of the $\psi \eta$ model. As in other tables of the text, the multiplicity, charges and the representation are shown for each set of fermions. $(\cdot)$ stands for a singlet representation.

discussed here. The consideration of the strong anomaly effective actions discussed here seems to lead us to clearer meaning of these comparisons, and with that, to point to a better understanding of the dynamics of strongly-coupled chiral gauge theories.

\section{Acknowledgments}

We thank Gabriele Veneziano for bringing [5] to our attention and for a comment, and David Tong for exchanges of views on some of the topics discussed here. The work is supported by the INFN special research initiative grant, "GAST" (Gauge and String Theories).

\section{A Chirally symmetric phase in the $\psi \eta$ model}

An interesting possibility for the $\psi \eta$ model is that no condensates form, the system confines and the flavor symmetry is unbroken [4]. The candidate massless degrees of freedom in the IR are $\frac{(N+4)(N+3)}{2}$ "baryons",

$$
B^{[A B]}=\psi^{\{i j\}} \eta_{i}^{A} \eta_{j}^{B}, \quad A, B=1,2, \ldots, N+4,
$$

antisymmetric in $A \leftrightarrow B$. All the $\mathrm{SU}(N+4) \times \mathrm{U}(1)$ anomalies are saturated by $B^{[A B]}$ as can be seen by using the data in table 1 . The discrete anomaly $\left(\mathbb{Z}_{N+2}\right)_{\psi}-[\mathrm{SU}(N)]^{2}$ is also matched as can be easily checked, and all other discrete anomalies are also matched as a consequence.

\section{B Color-flavor locked Higgs phase in the $\psi \boldsymbol{\eta}$ model}

Another possibility, in the $\psi \eta$ model, is that of a color-flavor locked phase [10, 16], with

$$
\left\langle\psi^{\{i j\}} \eta_{i}^{B}\right\rangle=C \Lambda^{3} \delta^{j B}, \quad j, B=1,2, \ldots N
$$

in which the symmetry is reduced to

$$
\mathrm{SU}(N)_{\mathrm{cf}} \times \mathrm{SU}(4)_{\mathrm{f}} \times \mathrm{U}(1)^{\prime}
$$

A subset of the same baryons $\left(B^{\left[A_{1} B_{1}\right]}\right.$ and $B^{\left[A_{1} B_{2}\right]}$ in the notation of table 2 ) saturate all of the triangles associated with the reduced symmetry group, see table 2 . The massless 


\begin{tabular}{|c|c|c|c|c|}
\hline & fields & $\mathrm{SU}(N)_{\mathrm{cf}}$ & $\mathrm{SU}(4)_{\mathrm{f}}$ & $U^{\prime}(1)$ \\
\hline UV & $\psi$ & $\square$ & $\frac{N(N+1)}{2} \cdot(\cdot)$ & 1 \\
& $\eta^{A_{1}}$ & $\square \oplus \bar{\boxminus}$ & $N^{2} \cdot(\cdot)$ & -1 \\
& $\eta^{A_{2}}$ & $4 \cdot \bar{\square}$ & $N \cdot \square$ & $-\frac{1}{2}$ \\
\hline \multirow{4}{*}{ IR } & $B^{\left[A_{1} B_{1}\right]}$ & $\bar{\boxminus}$ & $\frac{N(N-1)}{2} \cdot(\cdot)$ & -1 \\
& $B^{\left[A_{1} B_{2}\right]}$ & $4 \cdot \bar{\square}$ & $N \cdot \square$ & $-\frac{1}{2}$ \\
& $B^{\left[A_{2} B_{2}\right]}$ & $6 \cdot(\cdot)$ & $\boxminus$ & 0 \\
\hline
\end{tabular}

Table 2. Color-flavor locked phase in the $\psi \eta$ model. $A_{1}$ or $B_{1}$ stand for the first $N$ flavors $\left(A_{1}, B_{1}=1,2, \ldots, N\right)$, whereas $A_{2}$ or $B_{2}$ run over the rest of the flavor indices, $N+1, \ldots, N+4$. The set of potentially massless baryons $B^{\left[A_{2} B_{2}\right]}$, which were not explicitly taken into account in $[10,25]$, do not contribute to $\mathrm{SU}(N)_{\mathrm{cf}} \times \mathrm{SU}(4)_{\mathrm{f}} \times U^{\prime}(1)$ anomalies.

\begin{tabular}{|c|c|c|c|c|}
\hline & fields & $\mathrm{SU}(N)_{\mathrm{c}}$ & $\mathrm{SU}(N-4)$ & $\mathrm{U}(1)_{\chi \eta}$ \\
\hline $\mathrm{UV}$ & $\chi$ & $\bar{G}$ & $\frac{N(N-1)}{2} \cdot(\cdot)$ & $N-4$ \\
& $\eta^{A}$ & $(N-4) \cdot \square$ & $N \cdot \square$ & $-(N-2)$ \\
\hline IR & $B^{\{A B\}}$ & $\frac{(N-4)(N-3)}{2} \cdot(\cdot)$ & $\square$ & $-N$ \\
\hline
\end{tabular}

Table 3. Confinement and unbroken symmetry in the $\chi \eta$ model.

degrees of freedom are $\frac{N^{2}+7 N}{2}$ massless baryons $B^{\left[A_{1} B\right]}$ and $8 N+1 \mathrm{NG}$ bosons. To reproduce correctly the strong anomaly in the IR, however, another condensate $\langle B B\rangle \sim\langle\psi \eta \eta \psi \eta \eta\rangle$ and another set of massless baryon $B^{\left[A_{2} B_{2}\right]}$ (see table 2) are needed. This does not alter neither the symmetry breaking pattern (B.2), nor the anomaly matching. See section 3.2 for more discussion.

\section{Chirally symmetric phase in the $\chi \eta$ model}

Let us first examine the possibility that no condensates form, the system confines and the flavor symmetry is unbroken [3]. The massless baryons are

$$
B^{\{C D\}}=\chi_{[i j]} \eta^{i C} \eta^{j D}, \quad C, D=1,2, \ldots(N-4),
$$

symmetric in $C \leftrightarrow D$. The matching of the anomalies can be read off table 3 .

\section{Color-flavor locked Higgs vacuum in the $\chi \eta$ model}

It was pointed out [10] that this system may instead develop a condensate of the form

$$
\left\langle\chi_{[i j]} \eta^{B j}\right\rangle=C \Lambda^{3} \delta_{i}^{B}, \quad i, B=1,2, \ldots, N-4,
$$

namely,

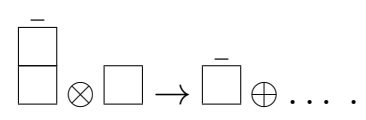




\begin{tabular}{|c|c|c|c|c|}
\hline & fields & $\mathrm{SU}(N-4)_{\mathrm{cf}}$ & $\mathrm{U}(1)^{\prime}$ & $\mathrm{SU}(4)_{\mathrm{c}}$ \\
\hline $\mathrm{UV}$ & $\chi_{i_{1} j_{1}}$ & $\bar{\theta}$ & $N$ & $\frac{(N-4)(N-5)}{2} \cdot(\cdot)$ \\
& $\chi_{i_{1} j_{2}}$ & $4 \cdot \bar{\square}$ & $\frac{N}{2}$ & $(N-4) \cdot \bar{\square}$ \\
& $\chi_{i_{2} j_{2}}$ & $\frac{4 \cdot 3}{2} \cdot(\cdot)$ & 0 & $\bar{\theta}$ \\
& $\eta^{i_{1}, A}$ & $\square \oplus \boxminus$ & $-N$ & $(N-4)^{2} \cdot(\cdot)$ \\
& $\eta^{i_{2}, A}$ & $4 \cdot \square$ & $-\frac{N}{2}$ & $(N-4) \cdot \square$ \\
\hline IR & $B^{\{A B\}}$ & $\square$ & $-N$ & $\frac{(N-4)(N-3)}{2} \cdot(\cdot)$ \\
\hline
\end{tabular}

Table 4. Color-flavor locking in the $\chi \eta$ model. The color index $i_{1}$ or $j_{1}$ runs up to $N-4$ and the rest is indicated by $i_{2}$ or $j_{2}$.

The symmetry is broken to

$$
\mathrm{SU}(N-4)_{\mathrm{cf}} \times \mathrm{SU}(4)_{\mathrm{c}} \times \mathrm{U}(1)^{\prime} .
$$

The massless baryons (C.1) saturate all the anomalies associated with $\mathrm{SU}(N-4)_{\mathrm{cf}} \times \mathrm{U}(1)^{\prime}$. There remains the $\chi_{i_{2} j_{2}}$ fermions which remain massless and strongly coupled to the $\mathrm{SU}(4)_{\mathrm{c}}$. We may assume that $\mathrm{SU}(4)_{\mathrm{c}}$ confines, and the condensate

$$
\langle\chi \chi\rangle \neq 0
$$

in

$$
\bar{\square} \otimes-\bar{\square} \rightarrow+\ldots,
$$

forms and $\chi_{i_{2} j_{2}}$ acquire dynamically mass. Assume that the massless baryons are:

$$
B^{\{A B\}}=\chi_{[i j]} \eta^{i A} \eta^{j B}, \quad A, B=1,2, \ldots(N-4),
$$

the saturation of all of the triangles associated can be seen in table 4 . The complementarity [40] apparently works here, in the sense that the massless sector of the dynamical Higgs phase has the same $\mathrm{SU}(N-4) \times \mathrm{U}(1)$ symmetry. See section 4 for more discussion.

\section{E Confining phase with unbroken global symmetries of the BY models}

The matter fields of the BY model are shown in table 5 .

The candidate massless composite fermions for the Bars-Yankielowitz models are the left-handed gauge-invariant fields:

$$
\left(B_{1}\right)^{[A B]}=\psi^{i j} \eta_{i}^{A} \eta_{j}^{B}, \quad\left(B_{2}\right)_{A}^{a}=\bar{\psi}_{i j} \bar{\eta}_{A}^{i} \xi^{j, a}, \quad\left(B_{3}\right)_{\{a b\}}=\psi^{i j} \bar{\xi}_{i, a} \bar{\xi}_{j, b}
$$

the first is anti-symmetric in $A \leftrightarrow B$ and the third is symmetric in $a \leftrightarrow b$; their charges are given in table 6 . Writing explicitly also the spin indices they are

$$
\begin{aligned}
\left(B_{1}\right)^{[A B], \alpha} & =\frac{1}{2} \epsilon_{\beta \gamma} \psi^{i j, \beta} \eta_{i}^{A, \gamma} \eta_{j}^{B, \alpha}+\frac{1}{2} \epsilon_{\beta \gamma} \psi^{i j, \beta} \eta_{i}^{A, \alpha} \eta_{j}^{B, \gamma}, \\
\left(B_{2}\right)_{A}^{a, \alpha} & =\epsilon_{\dot{\alpha} \dot{\beta}} \bar{\psi}_{i j}^{\dot{\alpha}} \bar{\eta}_{A}^{i, \dot{\beta}} \xi^{j, a, \alpha}, \quad\left(B_{3}\right)_{\{a b\}}^{\alpha}=\epsilon_{\dot{\beta} \dot{\gamma}} \psi^{i j, \alpha} \bar{\xi}_{i, a}^{\dot{\beta}} \bar{\xi}_{j, b}^{\dot{\gamma}}:
\end{aligned}
$$




\begin{tabular}{|c|c|c|c|c|c|}
\hline & $\mathrm{SU}(N)_{\mathrm{c}}$ & $\mathrm{SU}(N+4+p)$ & $\mathrm{SU}(p)$ & $\mathrm{U}(1)_{\psi \eta}$ & $\mathrm{U}(1)_{\psi \xi}$ \\
\hline$\psi$ & $\square$ & $\frac{N(N+1)}{2} \cdot(\cdot)$ & $\frac{N(N+1)}{2} \cdot(\cdot)$ & $N+4+p$ & $p$ \\
$\eta$ & $(N+4+p) \cdot \square$ & $N \cdot \square$ & $N(N+4+p) \cdot(\cdot)$ & $-(N+2)$ & 0 \\
$\xi$ & $p \cdot \square$ & $N p \cdot(\cdot)$ & $N \cdot \square$ & 0 & $-(N+2)$ \\
\hline
\end{tabular}

Table 5. The multiplicity, charges and the representation are shown for each set of fermions in the BY model.

\begin{tabular}{|c|c|c|c|c|c|}
\hline & $\mathrm{SU}(N)_{\mathrm{c}}$ & $\mathrm{SU}(N+4+p)$ & $\mathrm{SU}(p)$ & $\mathrm{U}(1)_{\psi \eta}$ & $\mathrm{U}(1)_{\psi \xi}$ \\
\hline$B_{1}$ & $\frac{(N+4+p)(N+3+p)}{2} \cdot(\cdot)$ & $\boxminus$ & $\frac{(N+4+p)(N+3+p)}{2} \cdot(\cdot)$ & $-N+p$ & $p$ \\
\hline$B_{2}$ & $(N+4+p) p \cdot(\cdot)$ & $p \cdot \bar{\square}$ & $(N+4+p) \cdot \square$ & $-(p+2)$ & $-(N+p+2)$ \\
\hline$B_{3}$ & $\frac{p(p+1)}{2} \cdot(\cdot)$ & $\frac{p(p+1)}{2} \cdot(\cdot)$ & $\square$ & $N+4+p$ & $2 N+4+p$ \\
\hline
\end{tabular}

Table 6. Chirally symmetric phase of the BY model.

\begin{tabular}{|c|c|c|}
\hline & UV & IR \\
\hline $\mathrm{SU}(N+4+p)^{3}$ & $N$ & $N+p-p$ \\
\hline $\mathrm{SU}(p)^{3}$ & $N$ & $N+4+p-(p+4)$ \\
\hline $\mathrm{SU}(N+4+p)^{2}-\mathrm{U}(1)_{\psi \eta}$ & $-N(N+2)$ & $-(N+2+p)(N-p)-p(p+2)$ \\
\hline $\mathrm{SU}(N+4+p)^{2}-\mathrm{U}(1)_{\psi \xi}$ & 0 & $(N+2+p) p-p(N+p+2)$ \\
\hline $\mathrm{SU}(p)^{2}-\mathrm{U}(1)_{\psi \eta}$ & 0 & $-(N+4+p)(p+2)+(p+2)(N+p+4)$ \\
\hline $\mathrm{SU}(p)^{2}-\mathrm{U}(1)_{\psi \xi}$ & $-N(N+2)$ & $-(N+4+p)(N+p+2)+(p+2)(2 N+p+4)$ \\
\hline $\mathrm{U}(1)_{\psi \eta}^{3}$ & $\frac{N(N+1)}{2}(N+4+p)^{3}-N(N+4+p)(N+2)^{3}$ & $\begin{array}{c}-\frac{(N+4+p)(N+3+p)}{2}(N-p)^{3}-(N+4+p) p(p+2)^{3}+ \\
+\frac{p(p+1)}{2}(N+4+p)^{3}\end{array}$ \\
\hline $\mathrm{U}(1)_{\psi \xi}^{3}$ & $\frac{N(N+1)}{2} p^{3}-N p(N+2)^{3}$ & $\begin{array}{c}\frac{(N+4+p)(N+3+p)}{2} p^{3}-(N+4+p) p(N+p+2)^{3}+ \\
+\frac{p(p+1)}{2}(2 N+4+p)^{3}\end{array}$ \\
\hline $\mathrm{Grav}^{2}-\mathrm{U}(1)_{\psi \eta}$ & $\frac{N(N+1)}{2}(N+4+p)-N(N+4+p)(N+2)$ & $\begin{array}{c}-\frac{(N+4+p)(N+3+p)}{2}(N-p)-(N+4+p) p(p+2)+ \\
+\frac{p(p+1)}{2}(N+4+p)\end{array}$ \\
\hline $\mathrm{Grav}^{2}-\mathrm{U}(1)_{\psi \xi}$ & $\frac{N(N+1)}{2} p-N p(N+2)$ & $\begin{array}{c}\frac{(N+4+p)(N+3+p)}{2} p-(N+4+p) p(N+p+2)+ \\
+\frac{p(p+1)}{2}(2 N+4+p)\end{array}$ \\
\hline $\mathrm{SU}(N+4+p)^{2}-\left(\mathbb{Z}_{N+2}\right)_{\psi}$ & 0 & $N+2+p-p=0 \bmod N+2$ \\
\hline $\mathrm{SU}(p)^{2}-\left(\mathbb{Z}_{N+2}\right)_{\psi}$ & 0 & $-(N+4+p)+p+2=0 \bmod N+2$ \\
\hline $\mathrm{Grav}^{2}-\left(\mathbb{Z}_{N+2}\right)_{\psi}$ & 1 & $1-1+1$ \\
\hline
\end{tabular}

Table 7. Anomaly matching checks for the IR chiral symmetric phase of the BY model. For $N$ odd, the last three equalities are consequences of other equations.

all transforming under the $\left\{\frac{1}{2}, 0\right\}$ representation of the Lorentz group. Table 7 summarizes the anomaly matching checks [25] via comparison between table 5 and table 6 .

\section{F Dynamical Higgs phase in the BY models}

The broken phase for the simplest of this class, the $\psi \eta$ model, has also been extensively in the main text, see also $[10,16,25,26]$. Something interesting happens for models with $p>0$ additional pairs of fermions in the fundamentals $(\eta, \xi)$. Now there is another channel, $\xi \eta$, which is gauge invariant and charged under the flavor group. We thus have 
a competition between two possible symmetry breaking channels, $\psi \eta$ and $\xi \eta$. We assume that both condensates occur in the following way:

$$
\begin{aligned}
\left\langle\psi^{i j} \eta_{i}^{B}\right\rangle & =c_{\psi \eta} \Lambda^{3} \delta^{j B} \neq 0, \quad j, B=1, \ldots, N, \\
\left\langle\xi^{i, a} \eta_{i}^{A}\right\rangle=c_{\eta \xi} \Lambda^{3} \delta^{a A} \neq 0, & a=1, \ldots, N, \quad A=N+1, \ldots, N+p
\end{aligned}
$$

where $\Lambda$ is the renormailization-invariant scale dynamically generated by the gauge interactions and $c_{\eta \xi}, c_{\psi \eta}$ are coefficients both of order one. According to the tumbling scenario [2], the first condensate to occur is in the maximally attractive channel (MAC). The strengths of the one-gluon exchange potential for the two channels

$$
\begin{array}{rrr}
\psi(\square) \eta(\square) & \text { forming } & \square \\
\xi(\square) \eta(\square) & \text { forming } & (\cdot),
\end{array}
$$

are, respectively,

$$
\begin{gathered}
\frac{N^{2}-1}{2 N}-\frac{(N+2)(N-1)}{N}-\frac{N^{2}-1}{2 N}=-\frac{(N+2)(N-1)}{N}, \\
0-2 \frac{N^{2}-1}{2 N}=-\frac{N^{2}-1}{N} .
\end{gathered}
$$

So the $\psi \eta$ channel is slightly more attractive, but such a perturbative argument is not really significant and we assume here that both types of condensates are formed.

The resulting pattern of symmetry breaking is

$$
\begin{aligned}
\mathrm{SU}(N)_{\mathrm{c}} \times \mathrm{SU}(N+4+p)_{\eta} \times \mathrm{SU}(p)_{\xi} \times \mathrm{U}(1)_{\psi \eta} \times \mathrm{U}(1)_{\psi \xi} \\
\stackrel{\langle\xi \eta\rangle,\langle\psi \eta\rangle}{\longrightarrow} \mathrm{SU}(N)_{\mathrm{cf}_{\eta}} \times \mathrm{SU}(4)_{\eta} \times \mathrm{SU}(p)_{\eta \xi} \times \mathrm{U}(1)_{\psi \eta}^{\prime} \times \mathrm{U}(1)_{\psi \xi}^{\prime} .
\end{aligned}
$$

At the end the color gauge symmetry is completely (dynamically) broken, leaving colorflavor diagonal $\mathrm{SU}(N)_{\mathrm{cf}_{\eta}}$ symmetry. $\mathrm{U}(1)_{\psi \eta}^{\prime}$ and $\mathrm{U}(1)_{\psi \xi}^{\prime}$ are combinations respectively of $\mathrm{U}(1)_{\psi \eta}$ and $\mathrm{U}(1)_{\xi \eta}$ with the element of $\mathrm{SU}(N+4+p)_{\eta}$ generated by

$$
t_{\mathrm{SU}(N+4+p)_{\eta}}=\left(\begin{array}{l|l|l}
(-\alpha(p+2)-p \beta) \mathbf{1}_{N \times N} & & \\
\hline & \frac{\alpha(N-p)-\beta p}{2} \mathbf{1}_{4 \times 4} & \\
\hline & & (\alpha+\beta)(N+2) \mathbf{1}_{p \times p}
\end{array}\right) .
$$

Making the decomposition of the fields in the direct sum of representations in the subgroup one gets table 8 . The composite massless baryons are subset of those in (3.36):

$$
\begin{array}{rlrl}
B_{1 a}^{[A B]} & =\psi^{i j} \eta_{i}^{A} \eta_{j}^{B}, & B_{1 b}^{[A C]} & =\psi^{i j} \eta_{i}^{A} \eta_{j}^{C}, \quad B_{1 c}^{[C D]}=\psi^{i j} \eta_{i}^{C} \eta_{j}^{D}, \\
A, B & =1, \ldots, N, \quad C, D=N+1, \ldots, N+4 .
\end{array}
$$

It is quite straightforward (see the remark in Introduction) to verify that the UV-IR anomaly matching continues to work, with the UV fermions in table 8 and the IR fermions in table 9 . 


\begin{tabular}{|c|c|c|c|c|c|}
\hline & $\mathrm{SU}(N)_{\mathrm{cf}_{\eta}}$ & $\mathrm{SU}(4)_{\eta}$ & $\mathrm{SU}(p)_{\eta \xi}$ & $\mathrm{U}(1)_{\psi \eta}^{\prime}$ & $\mathrm{U}(1)_{\psi \xi}^{\prime}$ \\
\hline$\psi$ & $\square$ & $\frac{N(N+1)}{2} \cdot(\cdot)$ & $\frac{N(N+1)}{2} \cdot(\cdot)$ & $N+4+p$ & $p$ \\
$\eta_{1}$ & $\square \oplus \bar{\boxminus}$ & $N^{2} \cdot(\cdot)$ & $N^{2} \cdot(\cdot)$ & $-(N+4+p)$ & $-p$ \\
$\eta_{2}$ & $4 \cdot \bar{\square}$ & $N \cdot \square$ & $4 N \cdot(\cdot)$ & $-\frac{N+p+4}{2}$ & $-\frac{p}{2}$ \\
$\eta_{3}$ & $p \cdot \bar{\square}$ & $N p \cdot(\cdot)$ & $N \cdot \bar{\square}$ & 0 & $N+2$ \\
$\xi$ & $p \cdot \square$ & $N p \cdot(\cdot)$ & $N \cdot \square$ & 0 & $-(N+2)$ \\
\hline
\end{tabular}

Table 8. UV fieds in the BY model, decomposed as a direct sum of the representations of the unbroken group of eq. (F.4).

\begin{tabular}{|c|c|c|c|c|c|}
\hline & $\mathrm{SU}(N)_{\mathrm{cf}_{\eta}}$ & $\mathrm{SU}(4)_{\eta}$ & $\mathrm{SU}(p)_{\eta \xi}$ & $\mathrm{U}(1)_{\psi \eta}^{\prime}$ & $\mathrm{U}(1)_{\psi \xi}^{\prime}$ \\
\hline$B_{1 a}$ & $\bar{G}$ & $\frac{N(N-1)}{2} \cdot(\cdot)$ & $\frac{N(N-1)}{2} \cdot(\cdot)$ & $-(N+4+p)$ & $-p$ \\
$B_{1 b}$ & $4 \cdot \bar{\square}$ & $N \cdot \square$ & $4 N \cdot(\cdot)$ & $-\frac{N+p+4}{2}$ & $-\frac{p}{2}$ \\
$B_{1 c}$ & $6 \cdot(\cdot)$ & $\boxminus$ & $6 \cdot(\cdot)$ & 0 & 0 \\
\hline
\end{tabular}

Table 9. IR massless fermions in the BY model in the Higgs phase.

As in the $\psi \eta$ model, the baryons indicated as $B_{1 c}$ in table 9 were not considered in the earlier work on the BY models [10, 25], but assumed here to be present and massless. These extra baryons do not contribute to the triangle anomalies with respect to unbroken symmetry group, see table 9, therefore do not affect the anomaly matching argument. However, as in the $\psi \eta$ model, the condensate $\left\langle B_{1 c} B_{1 c}\right\rangle \sim\langle\psi \eta \eta \psi \eta \eta\rangle$ is needed in order to reproduce the strong anomaly in the IR correctly. This however does not alter neither the symmetry breaking pattern (F.4), nor the conventional anomaly matching. See section 3.3 for more discussions.

\section{G Confining phase with unbroken global symmetries of the GG models}

The candidate massless composite fermions for the Georgi-Glashow models are:

$$
\left(B_{1}\right)^{\{A B\}}=\chi^{i j} \eta_{i}^{A} \eta_{j}^{B}, \quad\left(B_{2}\right)_{A}^{a}=\bar{\chi}_{i j} \bar{\eta}_{A}^{i} \xi^{j, a}, \quad\left(B_{3}\right)_{[a b]}=\chi^{i j} \bar{\xi}_{i, a} \bar{\xi}_{j, b}
$$

the first symmetric in $A \leftrightarrow B$ and the third anti-symmetric in $a \leftrightarrow b$. Writing the spin indices explicitly they are:

$$
\begin{aligned}
\left(B_{1}\right)^{\{A B\}, \alpha} & =\frac{1}{2} \epsilon_{\beta \gamma} \chi^{i j, \beta} \eta_{i}^{A, \gamma} \eta_{j}^{B, \alpha}+\frac{1}{2} \epsilon_{\beta \gamma} \chi^{i j, \beta} \eta_{i}^{A, \alpha} \eta_{j}^{B, \gamma}, \\
\left(B_{2}\right)_{A}^{a, \alpha} & =\epsilon_{\dot{\beta} \dot{\gamma}} \bar{\chi}_{i j}^{\dot{\beta}} \bar{\eta}_{\dot{A}}^{i, \dot{\gamma}} \xi^{j, a, \alpha}, \quad\left(B_{3}\right)_{[a b]}=\epsilon_{\dot{\beta} \dot{\gamma}} \chi^{i j} \bar{\xi}_{i, a}^{\dot{\beta}} \bar{\xi}_{j, b}^{\dot{\gamma}} .
\end{aligned}
$$

All anomaly triangles are saturated by these candidate massless composite fermions, see table 11. 


\begin{tabular}{|c|c|c|c|c|c|}
\hline & $\mathrm{SU}(N)_{\mathrm{c}}$ & $\mathrm{SU}(N-4+p)$ & $\mathrm{SU}(p)$ & $\mathrm{U}(1)_{\chi \eta}$ & $\mathrm{U}(1)_{\chi \xi}$ \\
\hline$B_{1}$ & $\frac{(N-4+p)(N-3+p)}{2} \cdot(\cdot)$ & $\square$ & $\frac{(N-4+p)(N-3+p)}{2} \cdot(\cdot)$ & $-N+p$ & $p$ \\
\hline$B_{2}$ & $(N-4+p) p \cdot(\cdot)$ & $p \cdot \bar{\square}$ & $(N-4+p) \cdot \square$ & $-(p-2)$ & $-(N+p-2)$ \\
\hline$B_{3}$ & $\frac{p(p-1)}{2} \cdot(\cdot)$ & $\frac{p(p-1)}{2} \cdot(\cdot)$ & $\bar{G}$ & $N-4+p$ & $2 N-4+p$ \\
\hline
\end{tabular}

Table 10. IR massless fermions in the chirally symmetric phase of the GG model.

\begin{tabular}{|c|c|c|}
\hline & UV & IR \\
\hline $\mathrm{SU}(N-4+p)^{3}$ & $N$ & $N+p-p$ \\
\hline $\mathrm{SU}(p)^{3}$ & $N$ & $N-4+p-(p-4)$ \\
\hline $\mathrm{SU}(N-4+p)^{2}-\mathrm{U}(1)_{\chi \eta}$ & $-N(N-2)$ & $-(N-2+p)(N-p)-p(p-2)$ \\
\hline $\mathrm{SU}(N-4+p)^{2}-\mathrm{U}(1)_{\chi \xi}$ & 0 & $(N-2+p) p-p(N+p-2)$ \\
\hline $\mathrm{SU}(p)^{2}-\mathrm{U}(1)_{\chi \eta}$ & 0 & $-(N-4+p)(p-2)+(p-2)(N-4+p)$ \\
\hline $\mathrm{SU}(p)^{2}-\mathrm{U}(1)_{\chi \xi}$ & $-N(N-2)$ & $-(N-4+p)(N+p-2)+(p-2)(2 N-4+p+0)$ \\
\hline $\mathrm{U}(1)_{\chi \eta}^{3}$ & $\frac{N(N-1)}{2}(N-4+p)^{3}-N(N-4+p)(N-2)^{3}$ & $\begin{array}{c}-\frac{(N-4+p)(N-3+p)}{2}(N-p)^{3}-(N-4+p) p(p-2)^{3}+ \\
+\frac{p(p-1)}{2}(N-4+p)^{3}\end{array}$ \\
\hline $\mathrm{U}(1)_{\chi \xi}^{3}$ & $\frac{N(N-1)}{2} p^{3}-N p(N-2)^{3}$ & $\begin{array}{c}\frac{(N-4+p)(N-3+p)}{2} p^{3}-(N-4+p) p(N+p-2)^{3}+ \\
+\frac{p(p-1)}{2}(2 N-4+p)^{3}\end{array}$ \\
\hline $\operatorname{Grav}^{2}-\mathrm{U}(1)_{\chi \eta}$ & $\frac{N(N-1)}{2}(N-4+p)-N(N-4+p)(N-2)$ & $\begin{array}{c}-\frac{(N-4+p)(N-3+p)}{2}(N-p)-(N-4+p) p(p-2)+ \\
+\frac{p(p-1)}{2}(N-4+p)\end{array}$ \\
\hline $\mathrm{Grav}^{2}-\mathrm{U}(1)_{\chi \xi}$ & $\frac{N(N-1)}{2} p-N p(N-2)$ & $\begin{array}{c}\frac{(N-4+p)(N-3+p)}{2} p-(N-4+p) p(N+p-2)+ \\
+\frac{p(p-1)}{2}(2 N-4+p)\end{array}$ \\
\hline $\mathrm{SU}(N-4+p)^{2}-\left(\mathbb{Z}_{N-2}\right)_{\chi}$ & 0 & $N-2+p-p=0 \bmod N-2$ \\
\hline $\mathrm{SU}(p)^{2}-\left(\mathbb{Z}_{N-2}\right)_{\chi}$ & 0 & $-(N-4+p)+p-2=0 \bmod N-2$ \\
\hline $\operatorname{Grav}^{2}-\left(\mathbb{Z}_{N-2}\right)_{\chi}$ & 1 & $1-1+1$ \\
\hline
\end{tabular}

Table 11. Anomaly matching checks for the IR chiral symmetric phase of the GG model.

\section{H Dynamical Higgs phase in the generalized GG models}

In the generalized Georgi-Glashow models there is a competition between two possible bifermion symmetry breaking channels $\chi \eta$ and $\xi \eta$. This time, the MAC criterion would slightly favor the $\xi \eta$ condensates against $\chi \eta$. Indeed, the strength of the one-gluon exchange potential for the two channels

$$
\begin{aligned}
& \chi(\square) \eta(\square) \quad \text { forming } \square \text {, } \\
& \xi(\square) \eta(\square) \quad \text { forming }(\cdot) \text {, }
\end{aligned}
$$

are, respectively,

$$
\begin{gathered}
\frac{N^{2}-1}{2 N}-\frac{(N-2)(N+1)}{N}-\frac{N^{2}-1}{2 N}=-\frac{(N-2)(N+1)}{N} \\
0-2 \frac{N^{2}-1}{2 N}=-\frac{N^{2}-1}{N}
\end{gathered}
$$




\begin{tabular}{|c|c|c|c|c|c|}
\hline & $\mathrm{SU}(N-4)_{\mathrm{cf}_{\eta}}$ & $\mathrm{SU}(4)_{\mathrm{c}}$ & $\mathrm{SU}(p)_{\eta \xi}$ & $\mathrm{U}(1)_{\chi \eta}^{\prime}$ & $\mathrm{U}(1)_{\chi \xi}^{\prime}$ \\
\hline$\chi_{1}$ & $\boxminus$ & $\frac{(N-4)(N-5)}{2} \cdot(\cdot)$ & $\frac{(N-4)(N-5)}{2} \cdot(\cdot)$ & $\frac{(N-4+p) N}{(N-4)}$ & $p \frac{N}{N-4}$ \\
$\chi_{2}$ & $4 \cdot \square$ & $(N-4) \cdot \square$ & $4(N-4) \cdot(\cdot)$ & $\frac{(N-4+p) N}{2(N-4)}$ & $\frac{p N}{2(N-4)}$ \\
$\chi_{3}$ & $6 \cdot(\cdot)$ & $\boxminus$ & $6 \cdot(\cdot)$ & 0 & 0 \\
$\eta_{1}$ & $\square \oplus \bar{\boxminus}$ & $(N-4)^{2} \cdot(\cdot)$ & $(N-4)^{2} \cdot(\cdot)$ & $-\frac{(N-4+p) N}{(N-4)}$ & $-\frac{p N}{N-4}$ \\
$\eta_{2}$ & $p \cdot \bar{\square}$ & $p(N-4) \cdot(\cdot)$ & $(N-4) \cdot \bar{\square}$ & $-2-2 \frac{p}{N-4}$ & $N-2-\frac{2 p}{N-4}$ \\
$\eta_{3}$ & $4 \cdot \bar{\square}$ & $(N-4) \cdot \bar{\square}$ & $4(N-4) \cdot(\cdot)$ & $-\frac{(N-4+p) N}{2(N-4)}$ & $-\frac{p N}{2(N-4)}$ \\
$\eta_{4}$ & $4 p \cdot(\cdot)$ & $p \cdot \bar{\square}$ & $4 \cdot \bar{\square}$ & $\frac{N-4+p}{2}$ & $N-2+\frac{p}{2}$ \\
$\xi_{1}$ & $p \cdot \square$ & $p(N-4) \cdot(\cdot)$ & $(N-4) \cdot \square$ & $2+2 \frac{p}{N-4}$ & $-(N-2)+\frac{2 p}{N-4}$ \\
$\xi_{2}$ & $4 p \cdot(\cdot)$ & $p \cdot \square$ & $4 \cdot \square$ & $-\frac{N-4+p}{2}$ & $-(N-2)-\frac{p}{2}$ \\
\hline
\end{tabular}

Table 12. UV fieds in the GG model, decomposed as a direct sum of the representations of the unbroken group of eq. (H.4).

Again, these perturbative estimates are not excessively significant, and we assume that both condensates occur as:

$$
\begin{aligned}
\left\langle\chi^{i j} \eta_{i}^{A}\right\rangle=c_{\chi \eta} \Lambda^{3} \delta^{j A} \neq 0, & j=1, \ldots, N-4, \quad A=1, \ldots, N-4, \\
\left\langle\xi^{i, a} \eta_{i}^{B}\right\rangle=c_{\eta \xi} \Lambda^{3} \delta^{a B} \neq 0, & a=1, \ldots, p, \quad B=N-4+1, \ldots, N-4+p .
\end{aligned}
$$

The pattern of symmetry breaking is

$$
\begin{aligned}
\mathrm{SU}(N)_{\mathrm{c}} & \times \mathrm{SU}(N-4+p)_{\eta} \times \mathrm{SU}(p)_{\xi} \times \mathrm{U}(1)_{\chi \eta} \times \mathrm{U}(1)_{\chi \xi} \\
& \stackrel{\langle\xi \eta\rangle,\langle\chi \eta\rangle}{\longrightarrow} \mathrm{SU}(4)_{\mathrm{c}} \times \mathrm{SU}(N-4)_{\mathrm{cf}_{\eta}} \times \mathrm{SU}(p)_{\eta \xi} \times \mathrm{U}(1)_{\chi \eta}^{\prime} \times \mathrm{U}(1)_{\chi \xi}^{\prime} .
\end{aligned}
$$

The color gauge symmetry is partially (dynamically) broken, leaving color-flavor diagonal global $\mathrm{SU}(N-4)_{\mathrm{cf}_{\eta}}$ symmetry and an $\mathrm{SU}(4)_{\mathrm{c}}$ gauge symmetry. $\mathrm{U}(1)_{\chi \eta}^{\prime}$ and $\mathrm{U}(1)_{\chi \xi}^{\prime}$ are a combinations respectively of $\mathrm{U}(1)_{\chi \eta}$ and $\mathrm{U}(1)_{\chi \xi}$ with the elements of $\mathrm{SU}(N)_{\mathrm{c}}$ and $\mathrm{SU}(N-4+p)_{\eta}$ generated by:

$$
\begin{aligned}
& t_{\mathrm{SU}(N)_{\mathrm{c}}}=\left(\begin{array}{l|l}
\frac{2 \frac{\alpha(N-4+p)+\beta p}{N-4} \mathbf{1}_{(N-4) \times(N-4)}}{} & \\
\hline & -\frac{\alpha(N-4+p)+\beta p}{2} \mathbf{1}_{4 \times 4}
\end{array}\right), \\
& t_{\mathrm{SU}(N-4+p)_{\eta}}=\left(\begin{array}{l|l}
-\frac{p(\alpha+\beta)(N-2)}{N-4} \mathbf{1}_{(N-4) \times(N-4)} \mid \\
\hline & (\alpha+\beta)(N-2) \mathbf{1}_{p \times p}
\end{array}\right) .
\end{aligned}
$$

Making the decomposition of the fields in the direct sum of representations in the subgroup one arrives at table 12 .

The composite massless baryons are subset of those in (G.1):

$$
B^{\{A B\}}=\chi^{i j} \eta_{i}^{A} \eta_{j}^{B}, \quad A, B=1, \ldots, N-4 .
$$

In the IR these fermions saturate all the anomalies of the unbroken chiral symmetry. This can be seen by an inspection of table 13 and table 12, with the help of the following observation. 


\begin{tabular}{|c|c|c|c|c|}
\hline & $\mathrm{SU}(N-4)_{\mathrm{cf}_{\eta}}$ & $\mathrm{SU}(p)_{\eta \xi}$ & $\mathrm{U}(1)_{\chi \eta}^{\prime}$ & $\mathrm{U}(1)_{\chi \xi}^{\prime}$ \\
\hline$B$ & $\square$ & $\frac{(N-4)(N-3)}{2} \cdot(\cdot)$ & $-\frac{(N-4+p) N}{(N-4)}$ & $-\frac{p N}{N-4}$ \\
\hline
\end{tabular}

Table 13. IR fied in the GG model in the dynamical Higgs phase.

In fact, there is a novel feature in the GG models, which is not shared by the BY models. As seen in table 13, there is an unbroken strong gauge symmetry $\mathrm{SU}(4)_{\mathrm{c}}$, with a set of fermions,

$$
\chi_{3}, \quad \chi_{2}, \quad \eta_{3}, \quad \eta_{4}, \quad \xi_{2}
$$

charged with respect to it. However, the pairs $\left\{\chi_{2}, \eta_{3}\right\}$ and $\left\{\eta_{4}, \xi_{2}\right\}$ can form massive Dirac fermions and decouple. These are vectorlike with respect to the surviving infrared symmetry, (H.4), hence are irrelevant to the anomalies. ${ }^{14}$ On the other hand, the fermion $\chi_{3}$ can condense

$$
\left\langle\chi_{3} \chi_{3}\right\rangle
$$

forming massive composite mesons, $\sim \chi_{3} \chi_{3}$, which also decouples. It is again neutral with respect to all of

$$
\mathrm{SU}(N-4)_{\mathrm{cf}_{\eta}} \times \mathrm{SU}(p)_{\eta \xi} \times \mathrm{U}(1)_{\chi \eta}^{\prime} \times \mathrm{U}(1)_{\chi \xi}^{\prime} .
$$

To summarize, $\mathrm{SU}(4)_{\mathrm{c}}$ is invisible (confines) in the IR, and only the unpaired part of the $\eta_{1}$ fermion $(\square)$ remains massless, and its contribution to the anomalies is reproduced exactly by the composite fermions, (H.6).

Comment: the massive mesons $\left\{\chi_{2} \eta_{3}\right\},\left\{\eta_{4} \xi_{2}\right\},\left\{\chi_{3} \chi_{3}\right\}$ are not charged with respect to the flavor symmetries surviving in the infrared. It is tempting to regard them as a toymodel "dark matter", as contrasted to the fermions $B^{A B}$ which constitute the "ordinary, visible" sector.

Open Access. This article is distributed under the terms of the Creative Commons Attribution License (CC-BY 4.0), which permits any use, distribution and reproduction in any medium, provided the original author(s) and source are credited.

\section{References}

[1] G. 't Hooft, Naturalness, chiral symmetry, and spontaneous chiral symmetry breaking, in Recent developments in gauge theories, G. 't Hooft et al. eds., Plenum Press, New York, U.S.A. (1980), reprinted in Dynamical Symmetry Breaking, E. Farhi et al. eds., World Scientific, Singapore (1982) and in Under the spell of the gauge principle, G. 't Hooft et al. eds., World Scientific, Singapore (1994).

[2] S. Raby, S. Dimopoulos and L. Susskind, Tumbling gauge theories, Nucl. Phys. B 169 (1980) 373 [INSPIRE].

\footnotetext{
${ }^{14}$ Actually, with matter fermions (H.7) $\mathrm{SU}(4)_{\mathrm{c}}$ is asymptotically free only for $50-2 N-2 p>0$. If $50-2 N-2 p<0, \mathrm{SU}(4)_{\mathrm{c}}$ will remain weakly coupled in the infrared, but the fact that the fermions (H.7) do not contribute to the anomalies with respect to the remaining flavor symmetries (H.9) stays valid.
} 
[3] S. Dimopoulos, S. Raby and L. Susskind, Light composite fermions, Nucl. Phys. B 173 (1980) 208 [INSPIRE].

[4] I. Bars and S. Yankielowicz, Composite quarks and leptons as solutions of anomaly constraints, Phys. Lett. B 101 (1981) 159 [INSPIRE].

[5] G. Veneziano, Tumbling and the strong anomaly, Phys. Lett. B 102 (1981) 139 [InSPIRE].

[6] J. Goity, R.D. Peccei and D. Zeppenfeld, Tumbling and complementarity in a chiral gauge theory, Nucl. Phys. B 262 (1985) 95 [INSPIRE].

[7] E. Eichten, R.D. Peccei, J. Preskill and D. Zeppenfeld, Chiral gauge theories in the $1 / n$ expansion, Nucl. Phys. B 268 (1986) 161 [INSPIRE].

[8] C.Q. Geng and R.E. Marshak, Two realistic preon models with $S U(N)$ metacolor satisfying complementarity, Phys. Rev. D $3 \mathbf{5}$ (1987) 2278 [INSPIRE].

[9] T. Appelquist, A.G. Cohen, M. Schmaltz and R. Shrock, New constraints on chiral gauge theories, Phys. Lett. B 459 (1999) 235 [hep-th/9904172] [InSPIRE].

[10] T. Appelquist, Z.-y. Duan and F. Sannino, Phases of chiral gauge theories, Phys. Rev. D 61 (2000) 125009 [hep-ph/0001043] [INSPIRE].

[11] M. Shifman and M. Ünsal, On Yang-Mills theories with chiral matter at strong coupling, Phys. Rev. D 79 (2009) 105010 [arXiv:0808.2485] [INSPIRE].

[12] E. Poppitz and Y. Shang, Chiral lattice gauge theories via mirror-fermion decoupling: a mission (im)possible?, Int. J. Mod. Phys. A 25 (2010) 2761 [arXiv: 1003.5896] [INSPIRE].

[13] A. Armoni and M. Shifman, A chiral $\mathrm{SU}(N)$ gauge theory planar equivalent to super-Yang-Mills, Phys. Rev. D 85 (2012) 105003 [arXiv:1202.1657] [INSPIRE].

[14] Y.-L. Shi and R. Shrock, $A_{k} \bar{F}$ chiral gauge theories, Phys. Rev. D 92 (2015) 105032 [arXiv: 1510.07663] [INSPIRE].

[15] Y.-L. Shi and R. Shrock, Renormalization-group evolution and nonperturbative behavior of chiral gauge theories with fermions in higher-dimensional representations, Phys. Rev. D 92 (2015) 125009 [arXiv: 1509.08501] [inSPIRE].

[16] S. Bolognesi, K. Konishi and M. Shifman, Patterns of symmetry breaking in chiral QCD, Phys. Rev. D 97 (2018) 094007 [arXiv:1712.04814] [InSPIRE].

[17] S. Bolognesi and K. Konishi, Dynamics and symmetries in chiral $\mathrm{SU}(N)$ gauge theories, Phys. Rev. D 100 (2019) 114008 [arXiv:1906.01485] [InSPIRE].

[18] E. Witten, Current algebra theorems for the U(1) Goldstone boson, Nucl. Phys. B 156 (1979) 269 [INSPIRE].

[19] G. Veneziano, U(1) without instantons, Nucl. Phys. B 159 (1979) 213 [inSPIRE].

[20] C. Rosenzweig, J. Schechter and C.G. Trahern, Is the effective lagrangian for QCD a sigma model?, Phys. Rev. D 21 (1980) 3388 [InSPIRE].

[21] P. Di Vecchia and G. Veneziano, Chiral dynamics in the large N limit, Nucl. Phys. B 171 (1980) 253 [INSPIRE].

[22] E. Witten, Large N chiral dynamics, Annals Phys. 128 (1980) 363 [InSPIRE].

[23] K. Kawarabayashi and N. Ohta, The problem of $\eta$ in the large $N$ limit: effective lagrangian approach, Nucl. Phys. B 175 (1980) 477 [INSPIRE].

[24] P. Nath and R.L. Arnowitt, The U(1) problem: current algebra and the theta vacuum, Phys. Rev. D 23 (1981) 473 [INSPIRE]. 
[25] S. Bolognesi, K. Konishi and A. Luzio, Probing the dynamics of chiral $\mathrm{SU}(N)$ gauge theories via generalized anomalies, Phys. Rev. D 103 (2021) 094016 [arXiv:2101.02601] [InSPIRE].

[26] S. Bolognesi, K. Konishi and A. Luzio, Dynamics from symmetries in chiral $\mathrm{SU}(N)$ gauge theories, JHEP 09 (2020) 001 [arXiv:2004.06639] [INSPIRE].

[27] G. Veneziano and S. Yankielowicz, An effective lagrangian for the pure $N=1$ supersymmetric Yang-Mills theory, Phys. Lett. B 113 (1982) 231 [InSPIRE].

[28] T.R. Taylor, G. Veneziano and S. Yankielowicz, Supersymmetric QCD and its massless limit: an effective lagrangian analysis, Nucl. Phys. B 218 (1983) 493 [inSPIRE].

[29] I. Affleck, M. Dine and N. Seiberg, Dynamical supersymmetry breaking in four-dimensions and its phenomenological implications, Nucl. Phys. B 256 (1985) 557 [INSPIRE].

[30] K. Konishi, Anomalous supersymmetry transformation of some composite operators in SQCD, Phys. Lett. B 135 (1984) 439 [InSPIRE].

[31] K.-i. Konishi and K.-i. Shizuya, Functional integral approach to chiral anomalies in supersymmetric gauge theories, Nuovo Cim. A 90 (1985) 111 [INSPIRE].

[32] D. Amati, K. Konishi, Y. Meurice, G.C. Rossi and G. Veneziano, Nonperturbative aspects in supersymmetric gauge theories, Phys. Rept. 162 (1988) 169 [INSPIRE].

[33] S. Yamaguchi, 't Hooft anomaly matching condition and chiral symmetry breaking without bilinear condensate, JHEP 01 (2019) 014 [arXiv: 1811.09390] [INSPIRE].

[34] S. Bolognesi, K. Konishi and A. Luzio, Gauging 1-form center symmetries in simple $\mathrm{SU}(N)$ gauge theories, JHEP 01 (2020) 048 [arXiv: 1909.06598] [INSPIRE].

[35] M.M. Anber and E. Poppitz, Two-flavor adjoint QCD, Phys. Rev. D 98 (2018) 034026 [arXiv: 1805.12290] [INSPIRE].

[36] J. Giedt, R. Brower, S. Catterall, G.T. Fleming and P. Vranas, Lattice super-Yang-Mills using domain wall fermions in the chiral limit, Phys. Rev. D 79 (2009) 025015 [arXiv:0810.5746] [INSPIRE].

[37] G. Bergner, C. López and S. Piemonte, Study of center and chiral symmetry realization in thermal $\mathcal{N}=1$ super Yang-Mills theory using the gradient flow, Phys. Rev. D 100 (2019) 074501 [arXiv: 1902.08469] [INSPIRE].

[38] S. Piemonte, G. Bergner and C. López, Monte Carlo simulations of overlap Majorana fermions, Phys. Rev. D 102 (2020) 014503 [arXiv: 2005.02236] [INSPIRE].

[39] P.B. Smith, A. Karasik, N. Lohitsiri and D. Tong, On discrete anomalies in chiral gauge theories, arXiv:2106.06402 [INSPIRE].

[40] E.H. Fradkin and S.H. Shenker, Phase diagrams of lattice gauge theories with Higgs fields, Phys. Rev. D 19 (1979) 3682 [InSPIRE].

[41] L.F. Abbott and E. Farhi, Are the weak interactions strong?, Phys. Lett. B 101 (1981) 69 [INSPIRE].

[42] M.G. Alford, K. Rajagopal and F. Wilczek, QCD at finite baryon density: Nucleon droplets and color superconductivity, Phys. Lett. B 422 (1998) 247 [hep-ph/9711395] [INSPIRE].

[43] M.G. Alford, K. Rajagopal and F. Wilczek, Color flavor locking and chiral symmetry breaking in high density QCD, Nucl. Phys. B 537 (1999) 443 [hep-ph/9804403] [INSPIRE]. 